\title{
Homogeneous ice nucleation evaluated for several water models
}

\author{
J. R. Espinosa, E. Sanz, C. Valeriani and C. Vega \\ Departamento de Química Física, Facultad de Ciencias Químicas, \\ Universidad Complutense de Madrid, 28040 Madrid, Spain
}

(Dated: November 26, 2014)

\begin{abstract}
In this work we evaluate by means of computer simulations the rate for ice homogeneous nucleation for several water models such as TIP4P, TIP4P/2005,TIP4P/ICE and $\mathrm{mW}$ (following the same procedure as in J.A.C.S. 13515008 (2013)) in a broad temperature range. We estimate the iceliquid interfacial free-energy, and conclude that for all water models $\gamma$ decreases as the temperature decreases. Extrapolating our results to the melting temperature, we obtain a value of the interfacial free-energy between 25 and $32 \mathrm{mN} / \mathrm{m}$ in reasonable agreement with the reported experimental values. Moreover, we observe that the values of $\gamma$ depend on the chosen water model and this is a key factor when numerically evaluating nucleation rates, given that the kinetic prefactor is quite similar for all water models with the exception of the $\mathrm{mW}$ (due to the absence of hydrogens). Somewhat surprisingly the estimates of the nucleation rates found in this work for TIP4P/2005 are slightly higher than those of the $\mathrm{mW}$ model, even though the former has explicit hydrogens. Our results suggest that it may be possible to observe spontaneous crystallization of TIP4P/2005 at about $60 \mathrm{~K}$ below the melting point.
\end{abstract}

\section{INTRODUCTION}

When liquid water is super-cooled to below its melting point, it becomes metastable and eventually freezes into its thermodynamically stable phase (ice). On the one hand, in the presence of impurities, this phase transition occurs quite easily (this is the reason why ice will appear in your refrigerator only after few hours). On the other hand, in the absence of impurities, metastable liquid water can survive even at temperatures well below the melting point, until homogeneous nucleation takes place and water is transformed into ice. Homogeneous nucleation is an activated process, given that the system has to overcome a nucleation free-energy barrier and to form a critical ice cluster in order to crystallize [1].

By performing experiments with micrometer-size water droplets, it has been possible to prepare metastable liquid water at temperatures down to $235 \mathrm{~K}$ [2 [5]. Below this temperature (known as the homogeneous nucleation temperature) water freezes in a few seconds. Such experiments permits one to experimentally determine the nucleation rate $\mathrm{J}$ (i.e the number of ice critical clusters per unit of volume and time) for temperatures between $235 \mathrm{~K}$ and $242 \mathrm{~K}$, with values of J defined within less than three orders of magnitude. Outside this range, it has not been possible to experimentally determine the nucleation rate, either because it is too large (below 235K) or too small (above 242K). Given that J is known only in a narrow temperature range, to estimate its values outside such range [5] classical nucleation theory (CNT) 1] could provide reasonable predictions, since the main ingredients needed are the interfacial free-energy of the liquid-ice interface at coexistence $(\gamma)$ and the kinetic prefactor. However, on the one hand, even though $\gamma$ could in principle be experimentally measured, its reported values (so far) range from 25 to $35 \mathrm{mN} / \mathrm{m}[\underline{6}]$; on the other hand, the kinetic prefactor is not known experimentally.

For these reasons, we believe that computer simula- tion could give a reasonable contribution in this context, since they could help both in determining the value of $\gamma$ and evaluating the homogeneous nucleation rate over a broader temperature range. As far as we are aware, little work has been devoted numerically to compute $\gamma$ for the ice-water interface: the only exception being Ref. [7, 8], where $\gamma$ was calculated at the melting point for several water models.

Moreover, work still needs to be done to estimate ice nucleation rates by means of numerical simulations. First of all, in order to know the amount of supercooling of liquid water (which determines the nucleation rate) one needs to know the melting temperature. However, until 2005 [9], the melting point of most water models had not been calculated. The first pioneering numerical paper on ice nucleation was that of Matsumoto et al [10], where spontaneous crystallization was observed at $230 \mathrm{~K}$ for a system of 500 molecules at a pressure of about -1000 bar using the TIP4P model [1]. Later on, for the same water model, the nucleation free-energy barrier had been calculated at $180 \mathrm{~K}$ in Ref. [12], [13] and [14]. The nucleation rate has been also recently computed for the $\mathrm{mW}$ water model[15] by Li et al [16] using forward flux sampling between $240 \mathrm{~K}$ and $220 \mathrm{~K}$, by Reinhardt and Doye 17] using umbrella sampling at $220 \mathrm{~K}$. At $220 \mathrm{~K}$ the value of $\mathrm{J}$ computed for $\mathrm{mW}$ by both groups differs by 5 orders of magnitude. This difference is somewhat larger than the expected statistical uncertainty for nucleation rates (which is expected to be of 1-2 orders of magnitude). Although both groups used different rare-events techniques the origin of the discrepancy it is not clear as for other systems the values of J computed from forward flux sampling and umbrella sampling seems to be in better agreement 18]. For the $\mathrm{mW}$ model using brute force simulations at 208K Moore and Molinero [19] were able to nucleate ice spontaneously in about 100ns in a system of 5000 molecules, leading to a rate of about $10^{32} \mathrm{~m}^{-3} \mathrm{~s}^{-1}$. In 2013 , our group estimated the value 
of $\mathrm{J}$ and $\gamma$ for other two water models, TIP4P/2005 20] and TIP4P/ICE 21, at low/moderate supercooling using the "seeding technique" 22, 23] together with CNT 24]. Even though the main advantage of the seeding technique is that it allows one to estimate the nucleation free-energy barrier even at moderate supercooling (differently from more rigorous numerical techniques such as umbrella sampling or forward flux sampling that might be CPU-time consuming at such temperatures), its main disadvantage is that it combines precise simulation results with an approximate theoretical formalism. The nucleation rates evaluated for both TIP4P/2005 and TIP4P/ICE water models 24] were in reasonable agreement with experiments. However, this agreement may have been due to a fortuitous cancellation of errors, occurring when an approximate water model is used in combination with an approximate technique. Therefore, in this work we will apply the same technique as in Ref. 24] to estimate ice nucleation rate using other water models, such as TIP4P [11] and $\mathrm{mW}$ [15].

In what follows, we will first provide more technical details about our previous work [24]. Next, we will analyze the differences in the estimates of $\gamma$ for several water models, and observe that values of $\gamma$ change significantly from a water model to another: even though for all water models $\gamma$ decreases as the temperature decreases. We then compute the kinetic prefactor, and conclude that it is quite similar for all water models, with the exception of $\mathrm{mW}$ for which it is about three orders of magnitude larger: this is certainly due to the lack of hydrogens in the model. However, being this difference significant, it is $\gamma$ that plays the central role in determining the nucleation rates. To conclude, we evaluate $\mathrm{J}$ and compare the results obtained for each water model. In particular we will focus on the $\mathrm{mW}$ model potential to determine whether the nucleation rate can be enhanced compared to other water models. We first observe that J estimated with the seeding technique compares nicely to the values of $\mathrm{J}$ reported for the same $\mathrm{mW}$ model in the literature (to within 5-6 orders of magnitude which is the expected uncertainty at high supercooling). Somewhat surprisingly, estimates of the nucleation rates for TIP4P/2005 are slightly higher than those for the mW model (even though the former has explicit hydrogens). The results of this work suggest that it may be possible to observe spontaneous crystallization of TIP4P/2005 at about 57K below the melting point (i.e. $195 \mathrm{~K}$ ). Given that nucleation rate at $230 \mathrm{~K}$ is very small, nucleation is not likely to be observed in computer simulations for TIP4P/2005. At such low temperature (and room pressure) a maximum in the compressibility has been found for this model by Abascal and Vega [25, 26] and Bresme et al. [27] thus providing a point of the Widom line. The results of this work support the existence of the Widom line for TIP4P/2005, and that this line is not due to the transient formation of ice [28].

\section{METHODOLOGY}

\section{A. The "seeding" technique}

The technique first proposed by Bai et al. [22, 23] consisted of inserting a solid cluster in a supercooled Lennard-Jones fluid, determining the temperature at which the cluster was critical (i.e where it can freeze or melt with equal probability). We shall denote this technique as "seeding" , as it can be regarded as the insertion of a seed of the stable phase (i.e the solid) in the supercooled liquid.

By assuming that classical nucleation theory can be used to describe and interpret the results obtained for the critical cluster size, then the technique allows one to estimate of the interfacial free energy $\gamma$ at the given thermodynamic conditions. According to CNT the critical cluster size $N_{c}$ is

$$
N_{c}=\frac{32 \pi \gamma^{3}}{3 \rho_{s}^{2}|\Delta \mu|^{3}}
$$

where $\rho_{s}$ is the number density of the solid phase (i.e ice Ih), $\Delta \mu$ the chemical potential difference between the solid and the fluid phase at the temperature at which the cluster is critical.

Once the value of $\gamma$ has been determined via Eq. 1 one can estimate (once again using CNT) the free energy barrier for nucleation from the expression :

$$
\Delta G_{c}=\frac{16 \pi \gamma^{3}}{3 \rho_{s}^{2}|\Delta \mu|^{2}} .
$$

Finally, one can estimate nucleation rates. Following the approach described in detail by Auer and Frenkel [1, 29, 30], J can be obtained from the expression :

$$
J=\rho_{f} Z f^{+} \exp \left(-\Delta G_{c} /\left(k_{B} T\right)\right)
$$

where $\left(\rho_{f} Z f^{+}\right)$is the kinetic prefactor, with $f^{+}$the attachment rate of particles to the critical cluster, $\rho_{f}$ the number density of the fluid and $Z$ the Zeldovich factor 1]. The CNT form of the Zeldovich factor is

$$
Z=\sqrt{\left(\left|\Delta G^{\prime \prime}\right|_{N_{c}} /\left(2 \pi k_{B} T\right)\right)}=\sqrt{|\Delta \mu| /\left(6 \pi k_{B} T N_{c}\right)}
$$

so that $\mathrm{Z}$ can be easily computed, once the size of the critical cluster $N_{c}$, the temperature at which it is critical $T$ and the chemical potential difference between the solid and the liquid are known. According to Ref. 229 31], $f^{+}$ can be computed as a diffusion coefficient of the cluster size at the top of the barrier ( at the temperature at which the cluster is critical):

$$
f^{+}=\frac{<\left(N(t)-N_{c}\right)^{2}>}{2 t} .
$$

The seeding technique can be particularly useful at moderate supercooling, where estimating the critical cluster 
size, the free-energy barrier height and the rate by more rigorous numerical techniques would be very CPU-time consuming.

A similar approach has been recently used by Pereyra et al 32], where the authors determined the temperature at which a cylindrical ice slab would melt or grow, in Ref. [33], by Knott et al determined the critical cluster size in a nucleation study of methane hydrate, and in Ref. 24], where Sanz et al studied ice nucleation from supercooled water.

\section{Drawbacks in the estimate of $\gamma$}

Admittedly, the way presented in Eq.(1) to estimate $\gamma$ is quite approximate, since it assumes that CNT is correct. The justification of this approach can be provided only a posteriori by comparison with more rigorous calculations.

First of all, different crystal planes will have different values of $\gamma$, whereas $\gamma$ computed according to Eq. (1) does not take into account the different crystal planes and only corresponds to an average among them. On the one hand, it has been shown for several systems such as hard spheres, Lennard Jones and water 7, 8, 14, 34 36] that comparing $\gamma$ computed for different planes results in differences smaller than about five per cent. On the other hand, one may assume that the spherical interface will represent the average value of $\gamma$ over different planes. In any case the relation between the value of $\gamma$ of a spherical cluster with that computed for a planar interface is not completely clear.

To conclude, our calculations of $\gamma$ rely on the assumption that the shape of the cluster is spherical. Visual inspection of our molecular dynamics trajectories suggests that this is indeed a reasonable approximation.

\section{B. Distinguishing between liquid and ice-like molecules}

As in our previous work 24], in order to identify molecules as liquid or ice Ih-like, we have used the $\bar{q}_{6}$ order parameter proposed in Ref. 37]: molecules with $\bar{q}_{6}$ larger than 0.358 will be classified as solid (ice Ih) and those with smaller values of as liquid-like.

Following this criterion, we conclude that only about 0.7 per cent of bulk ice Ih molecules are wrongly identified as liquid-like, and vice versa 0.7 per cent of bulk liquid molecules identified as solid-like (see Fig. 1). Since ice Ih and supercooled water have a quite similar structure, one may neglect this small mislabelling (furthermore it is very difficult to find order parameters with smaller mislabelling).

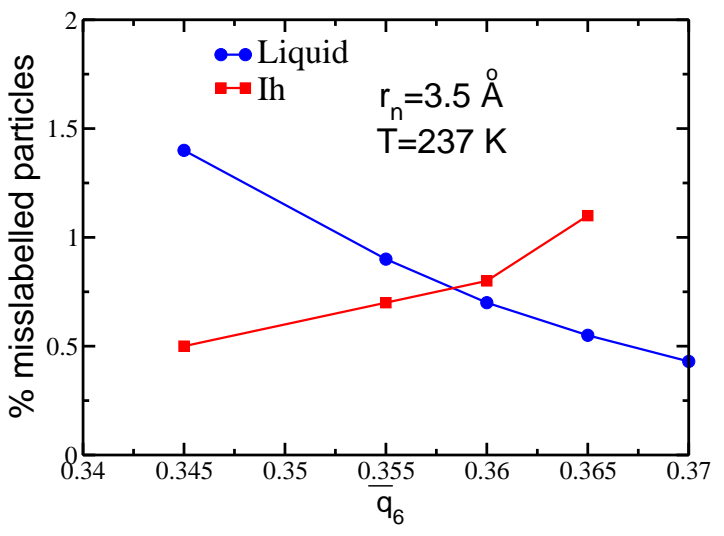

FIG. 1. Percentage of mislabelled particles according to $\bar{q}_{6}$, evaluated for bulk ice Ih and bulk liquid water at $237 \mathrm{~K}$ and 1bar (for TIP4P/2005), using the first minimum of the $g(r)$ as a cutoff for the calculation of $\bar{q}_{6}$. For ice Ih mislabelled particles are those with a value of the order parameter smaller than $\bar{q}_{6}$. For liquid water mislabelled particles are those with a value of the order parameter larger than $\bar{q}_{6}$.

Drawbacks in the estimate of $N_{c}$

The solid-fluid interface at the nucleus is not sharp, and we implicitly assume that the width of the interfacial region is very small relative to the size of the nucleus. This is of course, an approximation. Order parameters are very useful to distinguish between bulk ice and bulk liquid, but it is by far more difficult to distinguish between liquid and solid molecules in the interfacial region. [16 18] This constitutes a systematic source of error.

Therefore, determining $N_{c}$ entails an uncertainty due to the interfacial molecules. Of course, the larger the clusters the smaller the amount of uncertainty in $N_{c}$, since the ratio of the number of molecules at the interface to those in the cluster's core decreases with the system size. Whether the approach used in this work is reasonable or not can only be tested at posteriori, by comparing the results of this work with those found in the literature.

\section{Our setup for the seeding technique}

In this work, by means of the seeding technique, we determine the temperature at which three clusters of different sizes are critical. Three initial systems were obtained by inserting spherical ice Ih clusters of different sizes in supercooled water (molecules overlapping with the cluster were removed). After inserting the cluster, we equilibrated its interface for about $0.2 \mathrm{~ns}$ at $200 \mathrm{~K}$, enough to equilibrate the interface but not to observe melting or growing of the cluster (which typically requires 2 -20ns). After this $0.2 \mathrm{~ns}$ the sizes of the ice cluster were of about 7930, 3170 and 600 respectively for the three clusters sizes considered in this work. In each system, 
the total number of water molecules was about 20 times larger than the inserted cluster to avoid interactions between the cluster and its periodic images. Thus, the total number of molecules of water (considering both the ice Ih cluster and the molecules of the supercooled liquid ) were 182585,76781 and 22712 respectively. In order to be able to simulate such rather large systems, we had to recur to supercomputer facilities.

Once the cluster is equilibrated, we performed MD runs at different temperatures and monitor the cluster size to determine the temperature at which each cluster is critical.

There is an additional point worthy of comment concerning our initial setup. When implementing the seeding technique we use a starting cluster with Ih crystal structure. Yet in recent work, both experimental and numerical, have been strongly suggested that initial ice nuclei contain stacking faults. This has resulted in recent papers referring to stacking disordered ice I [38 43]. One may wonder about the consequences of this on the present study as it could have some impact on some relevant quantities such as the chemical potential difference, the interfacial free energy, and kinetic factors. This is an interesting point that deserves an independent study on its own. However there is some indication that the impact of the presence of stacking faults in ice I on the final results may be rather small. Free energy calculations ( obtained from the Einstein crystal calculations) for ices Ih and Ic using the TIP4P/2005 model indicate that the free energy difference between these two solid phases is quite small[44]. In addition, preliminary calculations similar to those performed in this work, but inserting a cluster of pure Ic, reveal little differences with those obtained using a cluster of ice Ih 45] ( suggesting that both the interfacial energy and the kinetic factors are quite similar for ices Ih and Ic).

\section{The chosen water model potentials}

In Ref. 24], we have studied both TIP4P/ICE and TIP4P/2005 water models, where MD runs were performed with Gromacs [46] using a velocity-rescaling [47] thermostat and an istropic Parrinello-Rahman barostat [48] with a relaxation time of about $2 \mathrm{ps}$. The LJ term of the potential was truncated at $9 \AA$ and long range corrections were added to account for the truncation of the LJ part. Ewald sums ( with the PME technique [49]) were used to deal with the electrostatic interactions. The real part of the electrostatic potential was also truncated at $9 \AA$. In this work we shall extend our previous study to the TIP4P model [11]. The details of the simulations are similar to those used in our previous work. In addition we have also performed simulations for the $\mathrm{mW}$ model of water [50]. Simulations for the $\mathrm{mW}$ model were performed using the LAMMPS package [51] In the mW water model hydrogens are not present, and tetrahedral ordering is induced by using three body forces. The model has no charges, and due to the short range of the two and three body forces it is computationally very fast.

The comparison between the results of TIP4P family models is of interest, as these models present the same charge distribution (with one LJ center on the oxygen, two positive charges on each $\mathrm{H}$ and a negative charge on the $\mathrm{H}-\mathrm{O}-\mathrm{H}$ bisector) but differ in the strength of the hydrogen bond (increasing as TIP4P, TIP4P/2005 and $\mathrm{TIP} 4 \mathrm{P} / \mathrm{ICE}$ ) and thus in their melting points (increasing in the same order).

The $\mathrm{mW}$ model has recently become quite popular in nucleation studies (either brute force [19] or using umbrella sampling or forward flux sampling techniques [16, 17]. Therefore, we will use this model to test the validity of the seeding technique and to analyze whether the absence of hydrogens speeds up the nucleation rate compared to other models where hydrogen atoms are explicit.

Let us finish this section with a final comment. In this work we are using classical statistical mechanics ( i.e standard molecular dynamics simulations). Since nucleation of ice occurs at low temperatures, where nuclear quantum effects gain importance, one may wonder about possible impact of such effects on nucleation studies of water. The parameters of empirical potentials are typically obtained by forcing the model to reproduce experimental properties within the framework of classical statistical mechanics. Thus the parameters of empirical potentials incorporate to some extend nuclear quantum effects in an effective way. That may explain the success of models like TIP4P/2005 to describe interfacial free energies and dynamic properties of real water. As will be shown in this work this strategy seems to also be successful when estimating nucleation rates of water. However, the properties of deuterated water (melting point, temperature of the maximum in density) differ significantly from those of water indicating that nuclear quantum effects are important and this effect can not be captured by classical statistical mechanics (i.e within this framework the melting point does not depend on the mass associated with the hydrogen atom). To capture isotopic effects in nucleation studies of water, it is necessary to have an accurate potential energy surface of water (obtained from accurate electronic structure calculations), and to incorporate nuclear quantum effects. However we have shown recently that by using a modified version of TIP4P/2005 (TIP4PQ/2005) in combination with path integral simulations, it is to describe reasonably well isotopic effects in water 52 55]. It would be interesting in the future to pursue a study similar to that performed in this work, where TIP4PQ/2005 is used in combination with path integral calculations to analyze isotopic effects on the nucleation of ice ( although this calculations would be at least one order of magnitude more expensive than those performed in this work). In any case the results of this work seem to indicate, that TIP4P/2005, in combination with classical simulations, seems to be reasonably successful in describing experimental values of the nu- 
cleation rates. Therefore the strategy of incorporating nuclear quantum effects via effective potentials does not seem too bad for this problem.

\section{RESULTS}

Before presenting our main results, we summarize a few properties at the melting point of the chosen water potentials (Table \).

TABLE I. Melting temperature, ice Ih density [56, 57], melting enthalpy and $\gamma$ at coexistence (extrapolated from the results for the finite size clusters) for TIP4P, TIP4P/2005, $\mathrm{TIP} 4 \mathrm{P} / \mathrm{ICE}, \mathrm{mW}$ and experiments.

\begin{tabular}{ccccc}
\hline model & $T_{m} / \mathrm{K}$ & $\rho_{s} /\left(\mathrm{gcm}^{-3}\right)$ & $\Delta H_{m} /(\mathrm{kcal} / \mathrm{mol})$ & $\gamma /(\mathrm{mN} / \mathrm{m})$ \\
\hline TIP4P & 230 & 0.94 & 1.05 & 25.6 \\
TIP4P $/ \mathrm{ICE}$ & 272 & 0.906 & 1.29 & 30.8 \\
$\mathrm{TIP} 4 \mathrm{P} / 2005$ & 252 & 0.921 & 1.16 & 29.0 \\
$\mathrm{~mW}$ & 274.6 & 0.978 & 1.26 & 29.6 \\
Experiment & 273.15 & 0.917 & 1.44 & 29 \\
\hline
\end{tabular}

All chosen water models differ in their properties at the melting point. No water model is able to simultaneously reproduce the coexistence density, the melting temperature and the melting enthalpy (even though TIP4P/ICE nicely reproduces the melting temperature and the solid density, it underestimates the melting enthalpy by about ten per cent). The experimental density of ice Ih at the melting point is $0.92 \mathrm{~g} / \mathrm{cm}^{3}[58]$. It is clear from the results of Table I that the density of ice Ih is very well reproduced by TIP4P/2005 and TIP4P/ICE, and reasonably well by TIP4P, whereas for $\mathrm{mW}$ the density of ice Ih is too high. Given that $\mathrm{mW}$ reproduces reasonably well the density of water at the melting point (i.e 1 $\mathrm{g} / \mathrm{cm}^{3}$ ) it turns out that for this water model the density change from ice Ih to liquid water is only of about 2 per cent, considerably smaller than that found in experiments where the density change is about 10 per cent. In other words, for $\mathrm{mW}$, freezing is a weakly first order phase transition.

Our main results for all water models are summarized in Table II. The runs used to determine the temperature at which each of the studied clusters becomes critical are provided as Supplementary Material [59].

\section{A. Ice Ih density}

As shown in Table II the density of ice Ih increases as the temperature decreases and this is also found in experiments ( at least up to $125 \mathrm{~K}$ ). Below this temperature the experimental density of ice $\mathrm{Ih}$ is approximately constant. This is a consequence of the third law of thermodynamics which implies that certain quantities such as the heat capacity or the the coefficient of thermal expansion tends to zero when the temperature goes to zero.
Since the coefficient of thermal expansion goes to zero at low temperatures that implies that the density of solid phases do not change much with temperature at low temperatures (at constant pressure). These effects can not be reproduced by classical simulations since their description would require the incorporation of nuclear quantum effects [52, 60]).

\section{B. The chemical potential difference between the fluid and the solid, $\Delta \mu$}

In order to determine the chemical potential difference between the liquid and the solid, we perform NpT simulations below melting for bulk ice Ih and liquid water. Next, we compute the enthalpy in both systems and perform thermodynamic integration to determine $\Delta \mu$ (at coexistence, the chemical potential of the solid and liquid are the same).
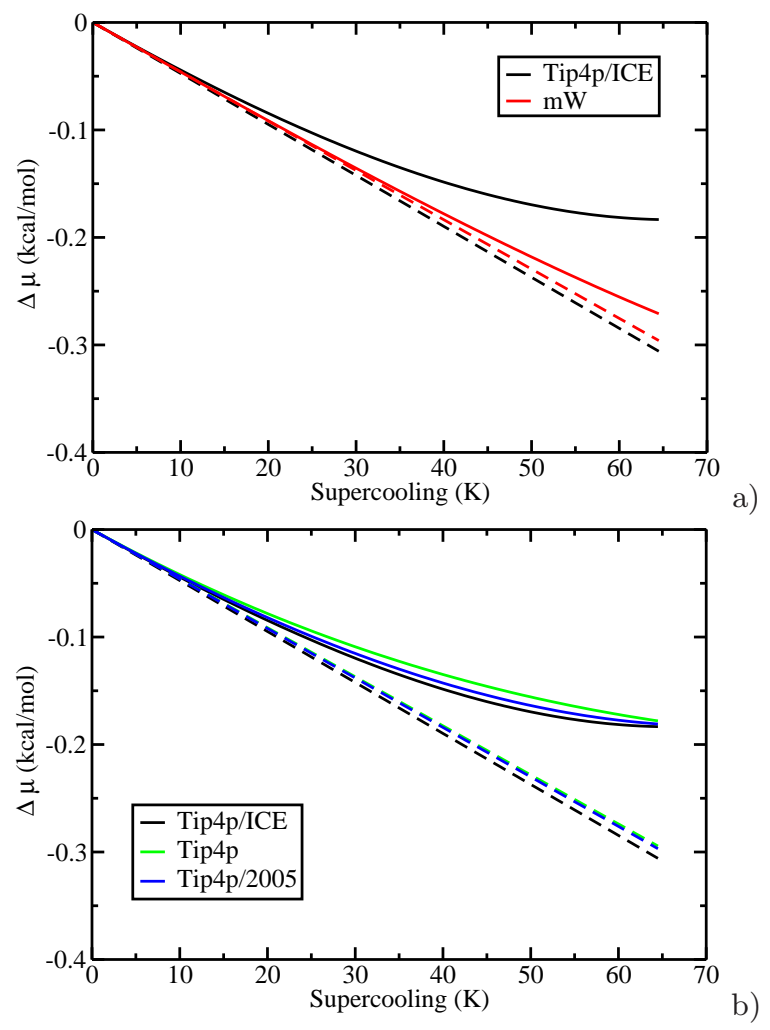

FIG. 2. $\Delta \mu$ obtained from thermodynamic integration (solid lines) and from Eq. 6 (dashed lines) as a function of the supercooling $\Delta T$. a) Results for $\mathrm{mW}$ and TIP4P/ICE. b) Results for TIP4P, TIP4P/2005 and TIP4P/ICE.

As shown in Fig 2, the value of $\Delta \mu$ is quite different for different models. $\Delta \mu$ can often be approximated using the enthalpy change at melting $[1]$ :

$$
\Delta \mu=\Delta H_{m}\left(1-\frac{T}{T_{m}}\right)
$$

As shown in Table I. the enthalpy change at melting 
TABLE II. Reported for a given cluster size and water model are the corresponding supercooling, $\Delta T / \mathrm{K}$, ice-Ih density, $\rho_{s} /\left(\mathrm{g} / \mathrm{cm}^{3}\right)$, chemical potential difference between the liquid and the solid, $\Delta \mu(\mathrm{kcal} / \mathrm{mol})$, number of particles in the cluster, $N_{c}$, attachment rate, $f^{+} / \mathrm{s}^{-1}$, Zeldovich factor, Z, diffusion coefficient, $\mathrm{D} /\left(\mathrm{m}^{2} / \mathrm{s}\right), \lambda / \AA$, interfacial free energy, $\gamma /(\mathrm{mN} / \mathrm{m})$, height of the nucleation free energy barrier, $\Delta G_{c} /\left(\mathrm{k}_{B} \mathrm{~T}\right)$, and decimal logarithm of the nucleation rate, $\log _{10}\left(\mathrm{~J} /\left(\mathrm{m}^{-3} \mathrm{~s}^{-1}\right)\right)$. Statistical errors for $\Delta G_{c}$ and $\log _{10}(J)$ are shown in parenthesis. The uncertainty in $\Delta T$ is of about $2.5 \mathrm{~K}$, so that the errors in $\Delta \mu, \gamma$ and $\Delta G_{c}$ are of about 7 per cent. As discussed in the main text, if systematic errors are included, the error in $\gamma$ does not increase much, but the error in $\Delta G_{c}$ and $\log _{10} J$ presented in this Table should be multiplied by two. For the medium clusters we have also included (in parenthesis) the value of the attachment rate and $\lambda$ obtained using only times larger than 1.5 ns in the determination of the attachment rate.

\begin{tabular}{|c|c|c|c|c|c|c|c|c|c|c|c|}
\hline Model & $\Delta T$ & $\rho_{s}$ & $\Delta \mu$ & $N_{c}$ & $f^{+}$ & $\mathrm{Z}$ & $\mathrm{D}$ & $\lambda$ & $\gamma$ & $\Delta G_{c}$ & $\log _{10} J$ \\
\hline Tip4p/ICE & 14.5 & 0.908 & 0.0629 & 7926 & $6.9 \cdot 10^{12}$ & $9.07 \cdot 10^{-4}$ & $1.80 \cdot 10^{-10}$ & 5.0 & 26.3 & $487(34)$ & $-173(16)$ \\
\hline Tip4p/ICE & 19.5 & 0.909 & 0.0826 & 3167 & $2.9(2.6) \cdot 10^{12}$ & $1.66 \cdot 10^{-3}$ & $9.63 \cdot 10^{-11}$ & $4.1(4.4)$ & 25.4 & $261(18)$ & $-75(9)$ \\
\hline Tip4p/ICE & 34.5 & 0.911 & 0.1335 & 600 & $3.0 \cdot 10^{11}$ & $5.00 \cdot 10^{-3}$ & $1.10 \cdot 10^{-11}$ & 2.2 & 23.7 & $85(6)$ & $1(4)$ \\
\hline Tip4p/2005 & 14.5 & 0.923 & 0.0612 & 7931 & $1.9 \cdot 10^{12}$ & $9.31 \cdot 10^{-4}$ & $1.48 \cdot 10^{-10}$ & 6.4 & 25.9 & $515(36)$ & $-186(17)$ \\
\hline Tip4p/2005 & 19.5 & 0.924 & 0.0801 & 3170 & $1.2(1.3) \cdot 10^{12}$ & $1.70 \cdot 10^{-3}$ & $9.69 \cdot 10^{-11}$ & 6.4 & 25.0 & $275(19)$ & $-83(9)$ \\
\hline Tip4p/2005 & 29.5 & 0.925 & 0.1137 & 600 & $1.8 \cdot 10^{11}$ & $4.76 \cdot 10^{-3}$ & $3.31 \cdot 10^{-11}$ & 6.5 & 20.4 & $77(5)$ & $3(3)$ \\
\hline Tip4p & 12.5 & 0.942 & 0.0515 & 7931 & $3.4 \cdot 10^{13}$ & $8.92 \cdot 10^{-4}$ & $1.44 \cdot 10^{-10}$ & 2.0 & 22.0 & $472(33)$ & $-166(15)$ \\
\hline Tip4p & 17.5 & 0.943 & 0.0696 & 3170 & $4.0(5.6) \cdot 10^{12}$ & $1.66 \cdot 10^{-3}$ & $4.90 \cdot 10^{-11}$ & 2.5 & 21.9 & $261(18)$ & $-75(9)$ \\
\hline Tip4p & 27.5 & 0.944 & 0.1018 & 600 & $1.8 \cdot 10^{11}$ & $4.73 \cdot 10^{-3}$ & $1.06 \cdot 10^{-11}$ & 3.1 & 18.5 & $76(5)$ & $4(3)$ \\
\hline $\mathrm{mW}$ & 14.6 & 0.980 & 0.0669 & 7926 & $9.0 \cdot 10^{14}$ & $9.32 \cdot 10^{-4}$ & $4.50 \cdot 10^{-9}$ & 2.2 & 29.5 & $514(36)$ & $-183(17)$ \\
\hline $\mathrm{mW}$ & 19.6 & 0.981 & 0.0895 & 3167 & $2.3(2.5) \cdot 10^{14}$ & $1.72 \cdot 10^{-3}$ & $2.33 \cdot 10^{-9}$ & 2.7 & 29.0 & $280(20)$ & $-81(9)$ \\
\hline $\mathrm{mW}$ & 34.6 & 0.983 & 0.1553 & 600 & $1.1 \cdot 10^{14}$ & $5.36 \cdot 10^{-3}$ & $2.69 \cdot 10^{-9}$ & 2.0 & 28.9 & $98(7)$ & $-2(4)$ \\
\hline
\end{tabular}

depends on the chosen model and at the same supercooling $\Delta \mu$ increases when using TIP4P, TIP4P/2005, TIP4P/ICE and $\mathrm{mW}$, respectively. Even though Eq. 6 allows one to explain the results of Fig. 2 for low supercoolings (where it becomes basically exact), it cannot be safely used for large supercoolings where the value of $\Delta \mu$ rigorously obtained from thermodynamic integration visibly differs from that obtained via Eq. 6. The reason for this difference is that the enthalpy of liquid water changes dramatically when water is supercooled, as shown by the increase in the heat capacity (which reaches a maximum at the so called Widom line [61]). The only water model where the approximation works is the $\mathrm{mW}$. For this model, the maximum in density is located at $250 \mathrm{~K}$, and the maximum in the heat capacity is displaced to lower temperatures. To conclude, the value of $\Delta \mu$ at large supercooling is sensitive to the thermodynamic behavior of supercooled water, and in particular to the location of the maximum in the heat capacity (if any) with respect to the melting temperature.

\section{Determining $N_{c}$}

To illustrate how the temperature at which the cluster is critical is determined we shall present one example for the $\mathrm{mW}$ model. In Fig 3 the time evolution of the cluster containing 600 ice molecules is shown for the mW model, at 1 bar and $\mathrm{T}=240 \mathrm{~K}$. At this temperature the cluster is critical and in approximately half of the trajectories it melts, whereas in the other half it grows.

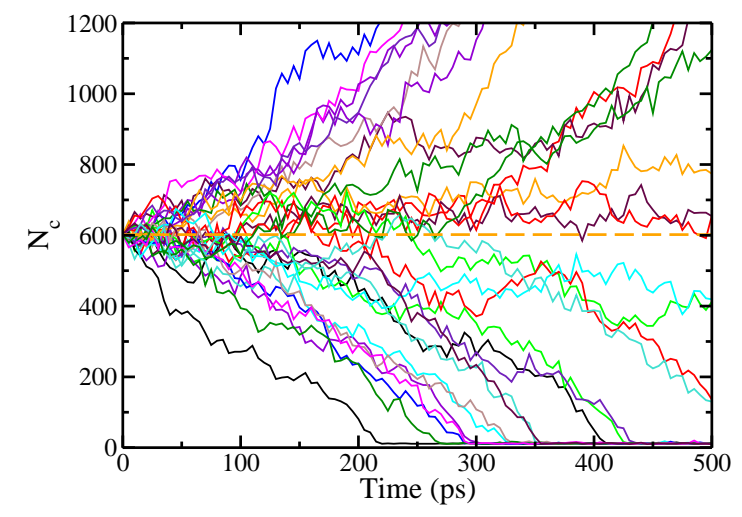

FIG. 3. Time evolution of the cluster size for the $\mathrm{mW}$ at $\mathrm{T}=240 \mathrm{~K}$ and 1 bar. The size of the initial cluster was about 600 molecules. Results obtained for 30 independent trajectories are shown.

\section{The interfacial free energy, $\gamma$}

By means of Eq1, we have estimated $\gamma$ for each cluster size. In Fig 4 the value of $\gamma$ is plotted as a function of the supercooling. As can be seen for all models $\gamma$ decreases with the temperature (i.e decreases as one increases the supercooling). Our results are compatible with a possible linear decrease of $\gamma$ with $\mathrm{T}$ although a faster (than linear) decreases of $\gamma$ with $\mathrm{T}$ cannot be discarded. The derivate of $\gamma$ with $\Delta T$ is the surface excess entropy. We obtained a slope of $-0.13,-0.25$ and $-0.38 \mathrm{mN} /$ (K.m) for TIP4P/ICE, TIP4P and TIP4P/2005 models. These slopes have large error bars arising from our uncertainty in the determina- 


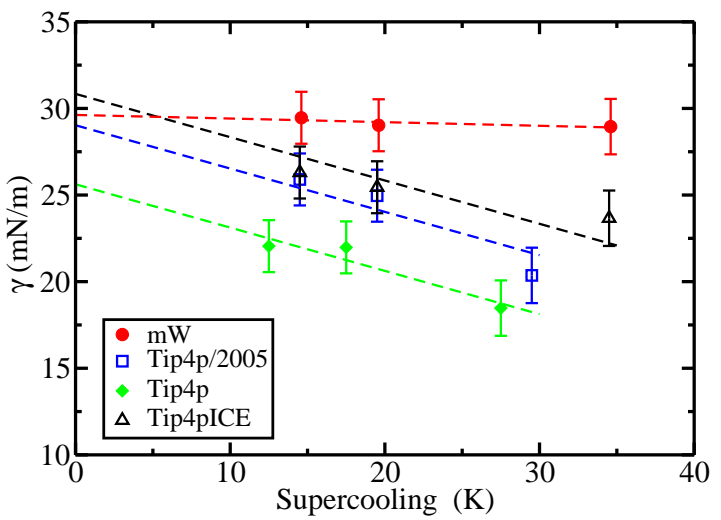

FIG. 4. Interfacial free energy between ice Ih and liquid water as a function of the degree of supercooling as obtained from the seeding technique in combination with CNT for TIP4P, TIP4P/2005, TIP4P/ICE and $\mathrm{mW}$.

tion of $\gamma$. To reduce such error bars we use the fact that all TIP4P-like models seem to display similar behavior, so we shall adopt the average slope, namely -0.25 $\mathrm{mN} /(\mathrm{K} . \mathrm{m})$, for the three models. Such slope is in good agreement with the slope calculated in Ref. 62] for the TIP4P/2005 (-0.18 mN/(K.m)). Experimentally there is no consensus neither on the value of $\gamma$ for the planar interface nor on the change of $\gamma$ with the degree of supercooling (see fig.10 in the paper of Pruppacher [2]). In any case the slope reported here, namely $-0.25 \mathrm{mN} /(K . \mathrm{m})$, is roughly consistent with the slopes presented in fig.10 of Ref. [2].

To estimate the value of $\gamma$ at the melting point we extrapolate our data to $\Delta T=0$ (using the averaged slope of $-0.25 \mathrm{mN} /(\mathrm{Km})$ for the TIP4 family models). The extrapolations are shown in Fig. 4 and the values of $\gamma$ at coexistence thus obtained are reported in Table [.

Within the TIP4P family the value of $\gamma$ increases with the strength of the hydrogen bond. Therefore within this family one could state that $\gamma$ increases with the melting enthalpy or with the melting point. The correlation between $\gamma$ and the melting enthalpy was first proposed by Turnbull [1, 63]. Another correlation between $\gamma$ and the melting point has been proposed by Laird [64]. We indeed confirm that for the TIP4P family both the correlation of Turnbull [63] and Laird 64] could be useful to predict the trends in $\gamma$. In fact, $\mathrm{mW}$ and TIP $4 \mathrm{P} / \mathrm{ICE}$ both have the same melting point and melting enthalpy. According to the Turnbull recipe, or the Laird recipe, they should have a quite similar value of $\gamma$. This seems to be consistent with the results of this work.

Moreover the results presented in Table円are in reasonable agreement with results obtained by other authors. Using the cleavage method and averaging over the basal, primary prismatic and secondary prismatic planes, the value of $\gamma$ for TIP4P, TIP4P-Ew 65] (a model with similar properties to TIP $4 \mathrm{P} / 2005)$ has been reported to be $26.5(4), 27.6(5) \mathrm{mJ} / \mathrm{m}^{2}$ respectively 7]. Using the $\mathrm{mW}$, Ref. [16] estimated $\gamma$ to be $31 \mathrm{mN} / \mathrm{m}$, in reasonable agree- ment with our estimate. Experimentally, the value of $\gamma$ for the ice Ih-water interface has been reported to be between 27 and $35 \mathrm{mN} / \mathrm{m}$. The most cited work is that of Ref.66] which reports a value of $29.1 \mathrm{mN} / \mathrm{m}$. In the absence of better criteria, we shall assume this to be the most reliable value. According to that, TIP4P/2005 provides estimates of $\gamma$ in agreement with experiments, TIP4P being slightly smaller than the experimental one, and the value of the $\mathrm{mW}$ and TIP4P/ICE slightly higher.

\section{E. The attachment rate, $f^{+}$}

When computing the attachment rate via Eq. 5] we observe that the results obtained for TIP4P, TIP4P/2005 and TIP4P/ICE are quite similar. The attachment rate is obtained after running 10 molecular dynamics trajectories at the temperature that makes the cluster critical (30 trajectories were performed in the case of the $\mathrm{mW}$ model ). In Fig. 5 we show (for the TIP4P model) the mean squared displacement (as obtained from the average of the 10 trajectories) of the cluster size as a function of time for the ice cluster of 3170 molecules (see also the Supplementary material [59]). All trajectories start from the same configuration and differ in the initial set of Maxwellian momenta. The results of Fig 5 were fitted to a straight line and the attachment rate is just half the value of the slope. The fact that we are starting all runs from the same configuration (although with different momenta) may have some impact on the computed slopes as pointed out recently by Rozmanov and Kusalik 67. This can be minimized by excluding the short-time behaviour from the calculation of the attachment rate. In Table II , we have determined the attachment rate for the medium cluster using both the entire window time and times larger than 1.5ns (results in parenthesis). As it can be seen, the impact on the attachment rate is small.

From the slope of the curve shown in Fig. 6] one can obtain $f^{+}$via Eq. 5. For the smallest cluster , $f^{+}$is of the order of $10^{11} s^{-1}$ whereas for the largest cluster is of the order of $10^{12}-10^{13} s^{-1}$. The results for the attachment rate $f^{+}$are shown in Table II. Notice that there was a misprint in the main text in our previous work 24 where we stated that the attachment rate for TIP4P/2005 of the medium cluster was $7010^{9} \mathrm{~s}^{-1}$. The correct value ( shown in Table III) is $1.2 \cdot 10^{12} \mathrm{~s}^{-1}$ and this correct value was used in the calculations of our previous work [24] leading to a value of $\log _{10} J$ of -83 which is the same as that reported here in Table II).

According to Ref. [1], since the attachment rate $f^{+}$, is related to the time required for a molecule to attach to the solid cluster, one could express it as

$$
f^{+}=\frac{24 D\left(N_{c}\right)^{2 / 3}}{\lambda^{2}}
$$

$N_{c}^{2 / 3}$ is the number of molecules at the cluster's surface and $\lambda^{2} / D$ is the time required for a molecule to diffuse 


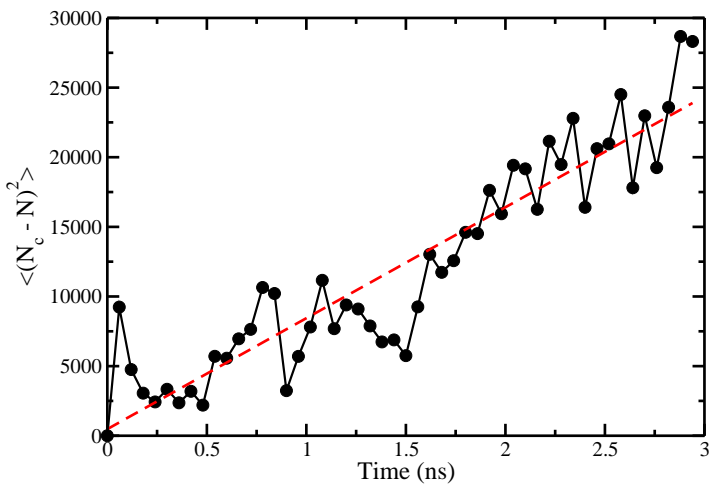

FIG. 5. Attachment rate for the cluster of 3170 molecules of the TIP4P model. Results obtained from the average of 10 different trajectories. Simulations were performed at $212.5 \mathrm{~K}$ and 1 bar. Notice that in Fig. 6 of our previous work 24], the results were obtained for the medium cluster of TIP4P/ICE and not for the medium cluster of the TIP4P/2005 model as stated in the caption.

a given length $\lambda$ ( $\mathrm{D}$ being the diffusion coefficient of the supercooled liquid phase). Having numerically computed $D$ at few temperatures, one could use an Arrhenius-like expression to estimate the diffusion coefficient as a function of temperature below melting:

$$
\ln D=\ln D_{0}-\frac{E_{a}}{R T}
$$

whose coefficients for each model are presented in Table III.

TABLE III. Coefficients of the fit of Eq 8 to the diffusion coefficient of supercooled water for the TIP4P, TIP4P/2005, TIP4P/ICE and $\mathrm{mW}$ water models.

\begin{tabular}{c|cc} 
Model & $\ln \left(D_{0} /\left(\mathrm{m}^{2} / \mathrm{s}\right)\right)$ & $E_{a} /(\mathrm{kJ} / \mathrm{mol})$ \\
\hline TIP4P & -1.30 & 39.526 \\
TIP4P $/ 2005$ & 2.88 & 50.803 \\
TIP4P $/ \mathrm{ICE}$ & -1.84 & 44.709 \\
$\mathrm{~mW}$ & -13.46 & 12.890 \\
\hline
\end{tabular}

Figure 6 clearly shows that an Arrhenius-like expression is sufficient to describe the variation of $\mathrm{D}$ with $\mathrm{T}$ for the temperature range considered in this work (i.e from the melting point up to temperatures of about $60 \mathrm{~K}$ below melting).

It is interesting to point out that $\mathrm{D}$ does not decrease much with temperature in the case of the $\mathrm{mW}$ model. The decrease of $\mathrm{D}$ with $\mathrm{T}$ is more pronounced in the case of the TIP4P potentials. In the figure we have also included experimental results [69]. As can be seen, the TIP4P/2005 model is able to describe the experimental values reasonably well. As shown in Fig.6 our values of $\mathrm{D}$ for TIP4P/2005 are entirely consistent with those determined previously ( for temperatures up to $210 \mathrm{~K}$ ) by Rozmanov and Kusalik 68].

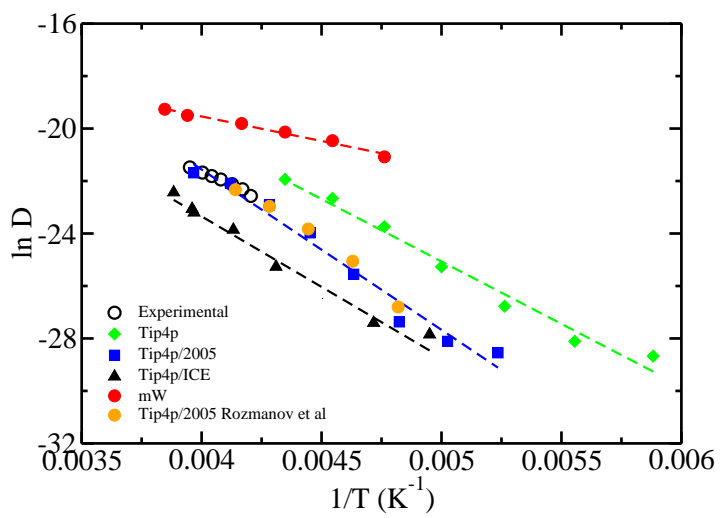

FIG. 6. The diffusion coefficients for TIP4P/2005, TIP4P/ICE, TIP4P and $\mathrm{mW}$ models. Symbols correspond to simulation results of this work. Lines were obtained from an Arrhenius fit. For the TIP4P/2005 model we have also included the results from Rozmanov and Kusalik 68] (orange circles) for temperatures up to $210 \mathrm{~K}$.

Experimental values: open circles [69].

Having determined the value of $\mathrm{D}$, we can estimate the value of $\lambda$ required to reproduce the results of $f^{+}$ obtained in this work using Eq. 7 (reported in Table I). The value of $\lambda$ (see Table III) is of about one molecule diameter (i.e $3.5 \AA$ ) and does not depend strongly neither on temperature nor on the water model. This means that in order to obtain fast and reasonable estimates of $f^{+}$ over a broad range of temperatures, one could in principle only need to determine $\mathrm{D}$, without having to recur to the expensive calculations needed to compute $f^{+}$using Eq 7 .

The attachment rate for the $\mathrm{mW}$ is about 2-3 orders of magnitude larger than that for the other models. Once again $\lambda$ is of the order of a molecular diameter. The larger value of $f^{+}$for the $\mathrm{mW}$ can be explained by taking into account the fact that for this model D is much larger than for the rest of the models ( and for real water) corresponding to an enhanced dynamics. Therefore we should point out that both $f^{+}$and $\mathrm{D}$ decreases with $\mathrm{T}$ much less in the $\mathrm{mW}$ model than in other models. The absence of explicit hydrogens provokes higher values of $\mathrm{D}, f^{+}$and faster dynamics. If the nucleation free-energy barrier of this model is similar to that of the other models considered in this work, then by considering the kinetic prefactor one should expect the kinetics of this model being three orders of magnitude faster than that of the other models.

\section{F. The kinetic prefactor}

The kinetic prefactor required to estimate $\mathrm{J}$ is given by the product of $\rho_{f}, \mathrm{Z}$ and $f^{+}$. The number density of the liquid, of the order of $10^{28}$ molecules $/ \mathrm{m}^{3}$, does not change much with temperature. The product $\mathrm{Zf}^{+}$does not have a strong temperature dependence either given that as the temperature decreases $\mathrm{Z}$ increases and $f^{+}$ 
decreases. Thus, we find that $\mathrm{Zf}^{+}$is of the order of $10^{9}$ $s^{-1}$ for the TIP4P family of models. Hence, the kinetic prefactor for TIP4P-like models is of the order of $10^{37}$ $\mathrm{m}^{-3} \mathrm{~s}^{-1}$.

As TIP4P/2005 describes quite well the diffusion coefficient of water at different temperatures, we believe that this is the order of magnitude of the kinetic prefactor of real water. Notice that for the $\mathrm{mW}$ model the kinetic prefactor is 2-3 orders of magnitude larger that for the TIP4P models. Therefore for the $\mathrm{mW}$ model the kinetic prefactor is of the order of $10^{40} \mathrm{~m}^{-3} \mathrm{~s}^{-1}$.

\section{G. The free-energy barrier, $\Delta G_{c}$}

The free-energy barriers for all clusters considered in this work are reported in Table II

For the largest clusters the free energy barrier is about $500 k_{B} \mathrm{~T}$, for the medium clusters about $250 k_{B} \mathrm{~T}$ and for the smallest clusters about $80 k_{B} \mathrm{~T}$ and the differences among models are not particularly large. The lowest value of the free-energy barrier corresponds to TIP4P and the largest to $\mathrm{mW}$ although the differences are not too large. For the $\mathrm{mW}$ model, which has a somewhat larger value of $\gamma$, one would expect the largest free-energy barriers. However, this is not the case given that both the ice density and $\Delta \mu$ are very large, partially compensating this effect.

For TIP4P/ICE our results differ from those of Ref. [70], where by means of umbrella sampling, a freeenergy barrier of $35 k_{B} T$ and a critical cluster of 300 molecules at a temperature of $235 \mathrm{~K}$ was reported. Our estimate is of $80 k_{B} T$ and 600 molecules at the same temperature. Performing 10 independent runs starting from an initial configuration of a 300 molecule cluster, we observed that the cluster always melted after 30-50ns. These results suggest that a cluster of 300 ice molecules is most likely sub-critical for these thermodynamic conditions. Although the order parameter used in Ref. 70] is different from that used in this work we found that both criteria differ only in about ten per cent in identifying the size of a given cluster.

We have included in Table II the statistical error in $\Delta G_{c}$. Once the order parameter is chosen then we can determine $N_{c}$ accurately ( so that there is practically no statistical error in the determination of $N_{c}$ ). We have an uncertainty of about $2.5 \mathrm{~K}$ in $\Delta T$, and that provokes an uncertainty of about $7 \%$ in both $\Delta \mu$ and $\gamma$. Notice that these two errors are not independent since we are obtaining $\gamma$ from Eq. 1. Therefore if $\Delta \mu$ is underestimated by $7 \%$ then $\gamma$ will be underestimated by $7 \%$ also. According to this the statistical error in $\Delta G_{c}$ is also about $7 \%$. The statistical error for $\Delta G_{c}$ is shown in Table II This statistical error can be reduced by performing more trajectories. In principle, this statistical error can be reduced at the expense of using a huge amount of CPU time.

There is however an additional source of uncertainty which is systematic and can not be reduced by performing more trajectories. Different order parameters will yield somewhat different values of $N_{c}$ (mainly due to the interfacial region). It is difficult to evaluate the impact of this systematic error (in fact if you know exactly the magnitude and sign of the systematic error you can always correct your results to the exact value !) and for this reason we shall just provide a rough estimate. Different (reasonable) order parameters gave differences of up to $N_{c}^{2 / 3}$ molecules for $N_{c}$. This gives a systematic error in $N_{c}$ of about $5 \%, 7 \%$ and $12 \%$ for the large, the medium and the small cluster respectively. of about $10 \%$. It follows then, that this systematic source of error would affect the values of $\gamma$ 1 by about $5 / 3 \%, 7 / 3 \%$ and $4 \%$ respectively. These systematic errors are smaller than the statistical error for $\gamma$ (of about $7 \%$ ). Since $\Delta G_{c}$ scales with $\gamma^{3}$ (see Eq. 2) then the systematic error would affect the values of $\Delta G_{c}$ by about $5 \% 7 \%$ and $12 \%$ respectively. We mentioned previously that the stochastic error in $\Delta G_{c}$ is of about $7 \%$. It seems that the systematic error for $\Delta G_{c}$ is similar to the stochastic error. In Table I we have included only the statistical errors in $\Delta G_{c}$. If one wishes to estimate the total error (i.e including the systematic error) one can roughly multiply the error of Table \by two.

\section{H. The nucleation rate, $\mathrm{J}$}

The homogeneous nucleation rate $\mathrm{J}$ is defined as the number of critical nuclei per unit of volume and time. Results of the nucleation rate are also reported in Table III. where we conclude that the order of magnitude changes from $10^{-180} \mathrm{~m}^{-3} \mathrm{~s}^{-1}$ for the temperatures around 15 degrees below melting to about $10^{0} \mathrm{~m}^{-3} \mathrm{~s}^{-1}$ for temperatures about $35 \mathrm{~K}$ below.

Due to the number of approximations we used to determine these numbers one might wonder whether our predictions for the nucleation rate $\mathrm{J}$ are reliable or not. The statistical error in $\log _{10} J$ is presented in Table II. The error in the kinetic prefactor in the expression of $\mathrm{J}$ has an error of about one order of magnitude. From the error in $\Delta G_{c}$ it is easy to obtain its contribution to the error in $\log _{10} J$ simply by dividing by 2.3 ( from the conversion natural to decimal logarithms). Therefore the total statistical error in $\log _{10} J$ is obtained after adding these two terms. As discussed previously, if systematic errors were also included then the error in $\log _{10} J$ presented in Table $\Pi$ should be (roughly) multiplied by two. From this it follows that the total error in $\log _{10} J$ (stochastic and systematic) is of about 40, 20 and 6 for the largest, medium and smallest clusters considered in this work.

We use the results obtained at three different temperatures to estimate $\mathrm{J}$ over a broad range of temperatures. For this purpose we need to calculate the height of the nucleation free energy barrier, $\Delta G_{c}$, and the kinetic prefactor, $Z \rho_{f} f^{+}$, for any temperature and obtain the rate 
with Eq. 3. To obtain $\Delta G_{c}(T)$ we use Eq. 2, where the functions $\gamma(T), \Delta \mu(T)$ and $\rho_{s}(T)$ are required. For $\gamma$, we assume that it changes linearly with $\mathrm{T}$ in the way shown by the fits in Fig. 4 (dashed lines). The chemical potential difference as a function of temperature is calculated by thermodynamic integration (see Fig. 2). The density of the solid as a function of temperature is taken from a linear fit to the results of Table III. To obtain the kinetic prefactor as a function of temperature we need $\rho_{f}(T), \mathrm{Z}(\mathrm{T})$ and $f^{+}(T)$. The density of the fluid changes smoothly with temperature and we have considered a constant value of $0.94 \mathrm{~g} / \mathrm{cm}^{3}$ for all models. By using Eqs. 4 and 1 and with the functions $\gamma(T)$ and $\Delta \mu(T)$ above described one can easily obtain $\mathrm{Z}(\mathrm{T})$. Finally, we use Eq. 7 to obtain $f^{+}(T)$. Eq. 7 requires, in turn, $\mathrm{D}(\mathrm{T})$ and $\lambda(T)$. For $\mathrm{D}(\mathrm{T})$ we use the fit given by Eq. 8 . For $\lambda$ we take a value independent of temperature and equal to an average between the values found for the three clusters (for all cases $\lambda$ is of the order of a molecular diameter). With these approximations (which appear quite reasonable after the results presented so far) we can obtain J for any value of $\Delta T$ (supercooling).

In fig:7 we present the results of the logarithm of J as a function of the degree of supercooling for different water models. In figure 7 (a) we show our results for the $\mathrm{mW}$ model and compare it with previous calculations of J. At $240 \mathrm{~K}$ our value of $\mathrm{J}$ is about 4 orders of magnitude higher that the value reported by $\mathrm{Li}$ et al.[16], whereas at $220 \mathrm{~K}$ , $215 \mathrm{~K}$ and $208 \mathrm{~K}$ our value is about $4-6$ orders of magnitude lower than the values reported by Li et al. 16. (220K using forward flux sampling), Russo et al.[71] (215K using umbrella sampling) and Moore and Molinero[19] (at 208K using brute force simulations). From this, we conclude that our predictions of the nucleation rate for the $\mathrm{mW}$ model are in reasonably good agreement with results previously reported in the literature, taking into account that the approach used here is an approximate one and that the uncertainty in $\mathrm{J}$ from our technique is about 6 orders of magnitude at high supercooling (coming from the uncertainty in determining the temperature at which the cluster is critical and the procedure used to distinguish solid from liquid-like molecules). We estimated the size of the critical cluster to be of 86 molecules at $205 \mathrm{~K}$ for the $\mathrm{mW}$ model, in excellent agreement with the value reported by Moore and Molinero for the same model and temperature which was of about 90 molecules [19]. Since Russo et al. 71] have determined not only nucleation rates but also, $f^{+}, N_{c}$ and the free energy barrier it is interesting to have a closer comparison term by term. This is done in Table IV As can be seen, the agreement for all individual terms is quite good. For $\log _{10}(J)$ is reasonable taking into account that, as discussed previously, our uncertainty in $\log _{10}(J)$ at high supercoolings, when all possible sources of error are considered, is of about 6 orders of magnitude.

Let us now describe the results for TIP4P like models. As it can be observed in figure $7 \mathrm{~b}$ ), the values of homogeneous nucleation rates for TIP4P/2005 almost co-
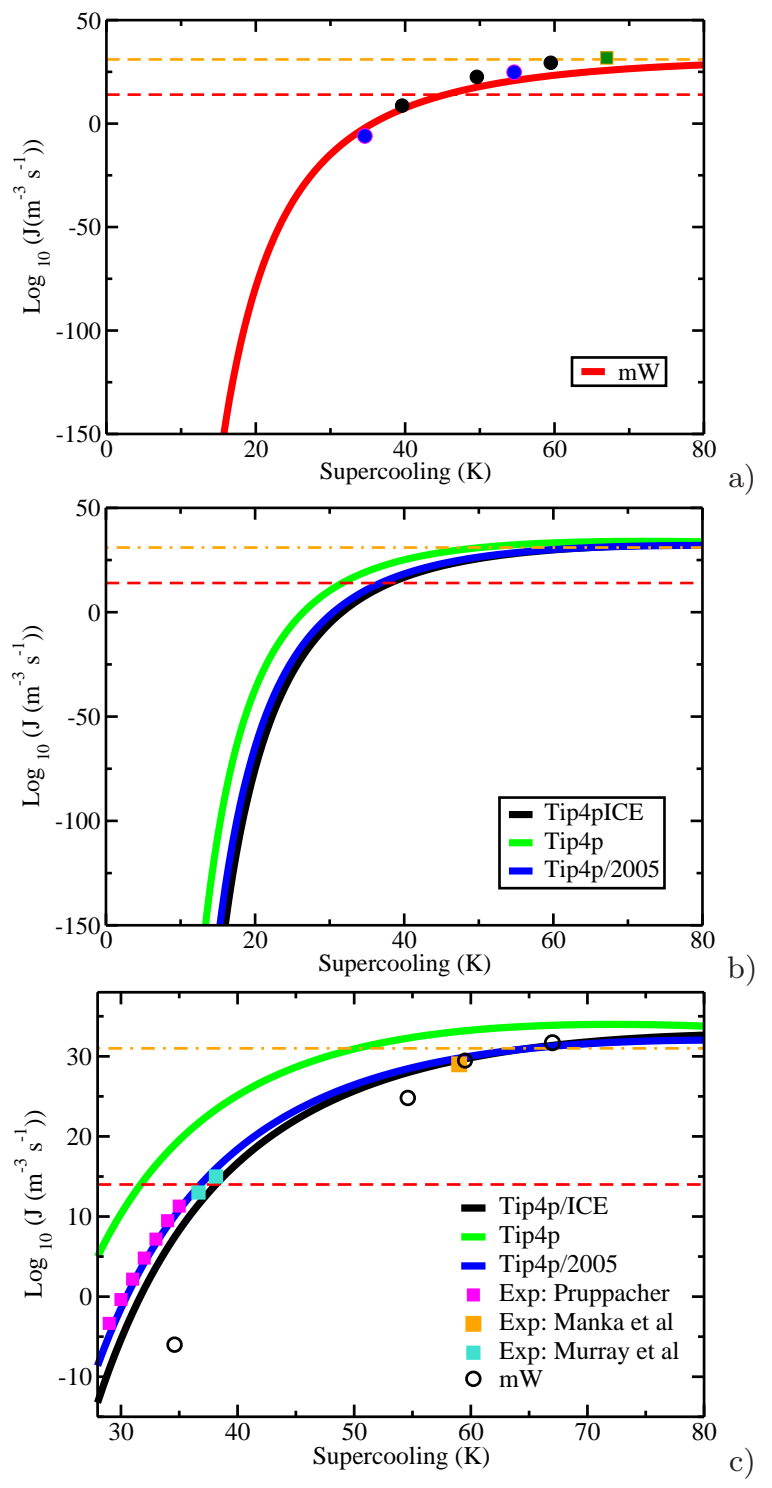

FIG. 7. Values of J for several water models, as obtained in this work, from experiment, and in previous work (in the case of the $\mathrm{mW}$ model). The horizontal lines correspond to $\log _{10} J /\left(m^{-3} s^{-1}\right)=14$ and $\log _{10} J /\left(m^{-3} s^{-1}\right)=31$ which are the approximate values of $\mathrm{J}$ at the homogeneous nucleation temperature in experiments and in simulations respectively. a) $\mathrm{J}$ for the $\mathrm{mW}$ model as obtained in this work ( red solid line). Blue circles are results at $240 \mathrm{~K}$ and $220 \mathrm{~K}$ from Ref. [16], black circles are the results from Russo et al. [71] and green square at $208 \mathrm{~K}$ from Ref. [19]. b) J for the TIP4P , TIP4P/ICE and TIP4P/2005 models. c) J of the models studied in this work (solid lines) compared to experiments (filled squares) of Pruppacher [2], Murray et al. 72] and Manka et al 73$]$. Empty circles are estimates of $\mathrm{J}$ for the $\mathrm{mW}$ model as reported in References [16, 19, 71]. Notice that, in the c panel, both $\mathrm{x}$ and $\mathrm{y}$-axis differ from the other two panels.

incide (to within the error bars) with those computed for TIP4P/ICE, whereas the ones for TIP4P are slightly higher. In Fig. $7 \mathrm{c}$ ) the values of homogeneous nucleation rates for TIP4P/2005 are compared to experimen- 
TABLE IV. Contributions (term by term) to $\mathrm{J}$ for the $\mathrm{mW}$ model as obtained in this work and as obtained by Russo et al. 71]. Results of this work were obtained at $p=1$ bar whereas those of Russo et al. [71] were obtained at $p=0 b a r$. This small difference of pressure is not expected to affect any of the terms of the table. $f^{+}$is given in $s^{-1}, \Delta G_{c}$ in $k_{B} T$ units and $\mathrm{J}$ in $\left(\mathrm{m}^{-3} \mathrm{~s}^{-1}\right)$

\begin{tabular}{ccccccc}
\hline Source & $\mathrm{T}(\mathrm{K})$ & $N_{c}$ & $f^{+}$ & $\mathrm{Z}$ & $\Delta G_{c}$ & $\log _{10}(J)$ \\
\hline \hline This work & 215.1 & 128 & $1.0 \times 10^{13}$ & 0.016 & 38.2 & 23.2 \\
Russo et al & 215.1 & 81 & $0.8 \times 10^{13}$ & 0.018 & 23.5 & 29.4 \\
\hline This work & 225 & 213 & $2.0 \times 10^{13}$ & 0.0109 & 51.5 & 17.5 \\
Russo et al & 225 & 180 & $2.6 \times 10^{13}$ & 0.0115 & 40.1 & 22.58 \\
\hline This work & 235 & 405 & $4.1 \times 10^{13}$ & 0.0070 & 76.3 & 6.8 \\
Russo et al & 235 & 400 & $4.7 \times 10^{13}$ & 0.0077 & 72.0 & 8.7 \\
\hline
\end{tabular}

tal ones at the temperatures where most experiments are available (i.e between $235 \mathrm{~K}$ and $240 \mathrm{~K}$ ). From the data we conclude that the results of TIP4P/2005 are consistent with the experimental ones ( taking into account the combined uncertainty of both experimental and simulation results). Thus it seems that TIP4P/2005 is able to reproduce not only the ice density, the ice-water interfacial free-energy and the diffusion coefficient, but also the nucleation rate $\mathrm{J}$ of real water. We also compare our results with recent experimental work where homogeneous ice nucleation was measured in nanoscopic water droplets [3]. By using such small droplets in Ref. 73] homogeneous ice nucleation was probed at an extremely high supercooling (59 $\mathrm{K}$ below melting). Notably, the agreement between the TIP4P/2005 model and the experiments of Ref. [73] is also very good. As can be seen in fig. $7 \mathrm{c}$ ) , for the $\mathrm{mW}$ model, the values of J obtained in previous works 16, 19, 71] seem to be in good agreement with the experimental results when the supercooling is large. In fact the agreement with the recent results of Manka et al. 73] is quite good. However for moderate supercooling J of the $\mathrm{mW}$ model seems to be lower than those found in experiments, this is most likely due to the high value of the interfacial free energy $\gamma$ of the model.

Another interesting feature is that for the TIP4P model the nucleation rate reaches a maximum value and after that it decreases slightly (see Fig. 7 c) . For the other TIP4P models one may expect similar behavior but at lower temperatures. The maximum is caused by the fact that the thermodynamic driving force for nucleation increases as the temperature decreases (i.e the free-energy barrier decreases) and at the same time the kinetic prefactor decreases dramatically with temperature and at very low temperatures becomes the dominant factor. The fact that $J$ may reach a maximum has been already suggested by Jeffery and Austin [74] and is consistent with the experimental results of Refs. [75, 76] when studying the freezing of water clusters at very low temperatures (i.e $72 \mathrm{~K}$ below melting), although is not entirely clear if at this high supercooling the formation of ice is limited by ice nucleation or by growth (see discussion below).

\section{Homogeneous nucleation temperature}

The homogeneous nucleation temperature $T_{H}$ is a kinetic concept. $T_{H}$ is the temperature below which water does not exist in its liquid phase (because it freezes). However, to properly define $T_{H}$, we need to specify both the sample size and the duration of the experiment. The experimental value of homogeneous nucleation temperature $\left(T_{H}^{e x p}=235 K\right)$ can be approximated by the temperature at which one critical ice cluster is formed in a spherical micrometer-size water droplet and for one minute:

$$
J=\frac{1}{\frac{4}{3} \pi\left(210^{-6}\right)^{3} 60}=10^{14} /\left(m^{3} s\right) .
$$

This experimental rate is represented by a dashed line in figure 7 As can be seen in figure $7 \mathrm{~b}$ ) in the case of TIP 4P/ICE and TIP4P/2005, $T_{H}^{e x p}$ is located about $37 \mathrm{~K}$ below melting, in reasonable agreement with the experimental value. In the case of TIP4P $T_{H}^{e x p}$ is slightly lower (around 30K below melting).

Let us now estimate the free-energy barrier height when $J=10^{14} /\left(\mathrm{m}^{3} \mathrm{~s}\right)$. For the TIP4P models it is of the order of $53 k_{B} \mathrm{~T}$ (given that the kinetic prefactor is about $10^{37} \mathrm{~m}^{-3} \mathrm{~s}^{-1}$ ) whereas for the $\mathrm{mW}$ model it is of the order of $60 k_{B}$ T. Since the values of J for TIP4P/2005 agree quite well with experiments, this strongly suggest that at the experimental value of $T_{H}^{\exp }$ (i.e $235 \mathrm{~K}$ ) the free energy barrier for nucleation is about $53 k_{B} \mathrm{~T}$. It is interesting to point out that the attachment rate $f^{+}$, of the $\mathrm{mW}$ model, is of the same order of magnitude of that found for LJ systems. Therefore for systems formed by atoms/ions,$f^{+}$seems to be of the same order magnitude. Obviously for these systems the free energy barrier must be about $60 k_{B} \mathrm{~T}$ at $T_{H}^{e x p}$ [1]. However, for water, $f^{+}$ is three orders of magnitude smaller and the free energy barrier at $T_{H}^{e x p}$ must be about $53 k_{B} \mathrm{~T}$. In other words as a rule of thumb one can state that the experimental homogeneous nucleation of water in micrometric droplets is the temperature at which the free energy barrier becomes of about $53 k_{B} T$. It is interesting to point out that both the value of the homogeneous nucleation temperature and of associated free energy barrier depend on the volume of the droplets with which the experiments are performed. The considerations above are all for micrometric water droplets, which is the most widespread experimental set up for the study of homogeneous ice nucleation. But this is not always the case. In fact, in a recent work, by using nanoscopic droplets much higher nucleation rates, and smaller nucleation barriers, were probed [73]. Therefore, the so called "homogeneous nucleation line" depends on the volume of the water droplets and should not be taken as a definite limit for the existence of supercooled liquid water. 
When dealing with computer simulations, both length and time-scales are quite different. The simulation value of the homogeneous nucleation temperature $\left(T_{H}^{s i m}\right)$ can be estimated as the temperature at which one critical ice cluster is formed in a simulation with a box side of $40 \AA$ (corresponding to a typical supercooled water density of about $0.94 \mathrm{~g} / \mathrm{cm}^{3}$ in a system of 2000 molecules) for 1 microsecond. At these conditions, the nucleation rate takes the value:

$$
J=\frac{1}{\left(4010^{-10}\right)^{3} 10^{-6}}=10^{31} /\left(m^{3} s\right)
$$

as represented by a dashed line in figure 7. As can be seen in figure $7 \mathrm{~b}$ ) in the case of TIP4P/ICE and TIP $4 \mathrm{P} / 2005, T_{H}^{s i m}$ is located about $60-65 \mathrm{~K}$ below melting. Whereas once more in the case of TIP4P $T_{H}^{\text {exp }}$ is slightly lower (around 50 below melting). Again, knowing the nucleation rate, one could estimate the free-energy barrier height at $T_{H}^{\text {sim }}$ for TIP4P/ICE, TIP4P/2005 and TIP4P to be of the order of $13 k_{B} \mathrm{~T}$. Whereas the freeenergy barrier height at $T_{H}^{s i m}$ for $\mathrm{mW}$ is of the order of $19 k_{B} \mathrm{~T}$ (given that the kinetic prefactor for this water model is three orders of magnitude larger). When simulating simple/atomic fluids (such as hard spheres [18], Lennard-Jones [77] or $\mathrm{NaCl}[31]$ ), spontaneous nucleation within reasonable time-scale can be observed with brute force simulations when the free-energy barrier height is of the order of $18 k_{B} \mathrm{~T}$.

\section{J. Growth rate and Avrami's law}

Rozmanov and Kusalik 78 have determined the growth rate of TIP4P/2005 for temperatures between the melting point and $210 \mathrm{~K}$ and fitted their results to a Wilson-Frenkel like expression 79 81]. To estimate the ice growth rate at lower temperatures we have performed direct coexistence simulations for the TIP4P/2005 at 1 bar and temperatures below $210 \mathrm{~K}$. The system consists of 2048 molecules. In the initial configuration half of the molecules are forming ice and the other half supercooled water (i.e approximately we have a $35 \AA$ layer of ice and a $35 \AA$ layer of water). The evolution of the potential energy with time is shown in fig, 8 . For all considered temperatures the system freezes completely ( as shown from the final plateau of the energy, from visual inspection of the final configuration and from the analysis of the sample using order parameters). Obviously the time required to form ice is much longer at $200 \mathrm{~K}$ (1500ns) than at $220 \mathrm{~K}$ (80ns). To estimate the growth rate we simply divided $35 \AA$ (i.e the thickness of the liquid slab) by the time required to freeze the system. Notice that this is used just to provide a rough estimate of the growth rate of ice. A rigorous determination of the growth rate requires performing the analysis over a larger number of independent trajectories. For the three highest temperatures $220 \mathrm{~K}, 215 \mathrm{~K}$ and $210 \mathrm{~K}$ the growth rate estimated in this work is fully consistent with that obtained pre- viously by Rozmanov and Kusalik. 78, 82] For the two lowest temperatures, the growth rate estimated in this work , $0.049 \AA / \mathrm{ns}$ at $205 \mathrm{~K}$ and $0.025 \AA /$ ns at $200 \mathrm{~K}$, should be compared to the values $0.056 \AA / \mathrm{ns}$ and 0.040 $\AA /$ ns obtained from the fit of Rozmanov and Kusalik (for the average of the different planes). 82] Since the agreement is satisfactory we shall assume that the fit of Rozmanov and Kusalik for the ice growth rate, can be used for temperatures below $210 \mathrm{~K}$.

In general, nucleation is the limiting step for supercooled liquid water to transform into ice. Therefore, once a critical cluster is formed, ice crystal growth tends to occur quite rapidly. However, at low temperatures this might not be the case, since the ice growth rate, $\mathrm{u}$, might be very small. When the growth rate is small, one should introduce a new parameter, $\tau_{x}$ (which is the time required to crystallize a certain volume fraction of the sample $\phi) . \tau_{x}$ depends on two properties, the value of $\mathrm{J}$, and the value of the growth rate of ice, $\mathrm{u}$. The Avrami's equation has been considered for obtaining $\tau_{x}$ [19, 83, 84]. In Debenedetti's book the expression for $\tau_{x}$ is provided and it is given by :

$$
\tau_{x}^{A v r a m i}=\left((3 \phi) /\left(\pi J u^{3}\right)\right)^{1 / 4}
$$

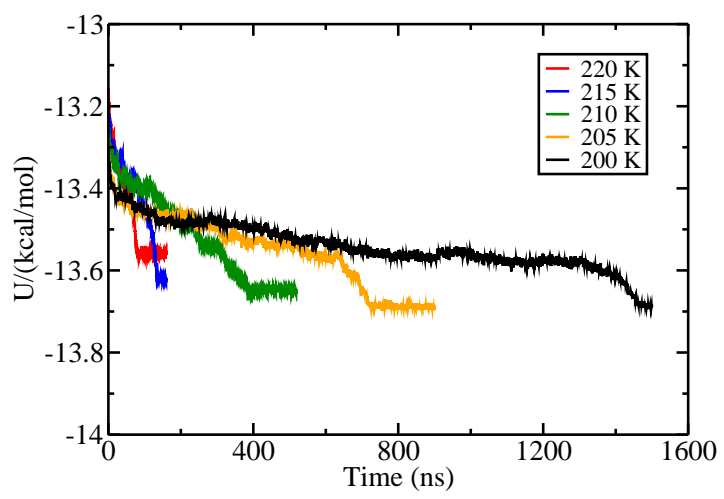

FIG. 8. Evolution of the potential energy with time in direct coexistence runs of the TIP4P/2005 model using a slab with 2048 molecules (half ice Ih and half liquid water) at the temperatures, from the left to the right, of $220 \mathrm{~K}, 215 \mathrm{~K}, 210 \mathrm{~K}$, $205 \mathrm{~K}$ and $200 \mathrm{~K}$.

By using Avrami's expression we have plotted $\tau_{x}^{\text {Avrami }}$ in fig. 9 as a function of the degree of supercooling for the TIP4P / 2005 model. Although we used $\phi=0.7, \tau_{x}^{\text {Avrami }}$ is practically the same for any value between 0.6 and 0.9 chosen for $\phi$, as $\tau_{x}$ changes as $\phi^{1 / 4}$. As can be seen the minimum $\tau_{x}$ is of the order of several microseconds. The minimum in $\tau_{x}$ occurs at smaller values of supercooling that the maximum in $\mathrm{J}$. In any case it is important to recall the fact that $\tau_{x}$ rather than $\mathrm{J}$ is the relevant magnitude at large supercoolings as the growth rate of ice can be the limiting factor. There is still a subtle issue with respect to the application of Avrami's expression. Notice that Avrami's expression contains only the intensive parameters $\phi, \mathrm{J}$, and $\mathrm{u}$, so $\tau_{x}$ does not depend on 
the size of the system. However, as pointed out by Berg [85], there are important system size effects on $\tau_{x}$ specially when one goes down to the system size typical of computer simulations. When the nucleation time $\tau_{n u}$ (i.e the time required to form a critical nucleus) :

$$
\tau_{n u}=1 /(J V)
$$

is larger than the diffusive time one can not find a critical cluster growing in the system until the nucleation time has elapsed. In such regime, Avrami's traditional expression can not be applied and the crystallization time is dominated by the nucleation time, that is inversely proportional to the system's volume. Following Berg [85], let us define a parameter $q$ as the ratio of two times, the growth time $\tau_{\text {growth }}$ (i.e the time required to crystallize completely the simulation box after a post-critical nucleus has been formed) and $\tau_{n u}$ as :

$$
\begin{gathered}
\tau_{\text {growth }}=L / u \\
q=\tau_{\text {growth }} / \tau_{n u}
\end{gathered}
$$

where $\mathrm{L}$ is the dimension of one of the sizes of the cubic simulation box. Notice that q depends on the system size so it is not an intensive property. In fact for any temperature $\mathrm{q}$ tends to $\infty$ as one increases the system size to the thermodynamic limit ( the numerator scales as $L$ whereas the denominator scales as $\left.L^{-3}\right)$. According to Berg [85] $\tau_{x}$ can be expressed as :

$$
\tau_{x}=\tau_{n u}\left(1+f_{d}(q)\right)
$$

The function $f_{d}(q)$ behaves as $A_{d} q$ for values of $q$ smaller than one (i.e when the growth time is smaller than the nucleation time) and behaves as $B_{d} q^{3 / 4}$ for values of $q$ larger than 64 (i.e when the growth time is larger than the nucleation time). Between these two values one has a crossover behavior. According to this for values of $q$ larger than 64 , one recovers the traditional Avrami's expression. However for small values of $\mathrm{q} \tau_{x}$ can be approximated quite well by $\tau_{n u}$. Since $\tau_{n u}$ depends on the system size, so does $\tau_{x}$. In fig. 9 we have also plotted the value of $\tau_{n u}$ for system sizes of 2000, 20000, 200000 and 2000000 molecules of water. For each system size $\tau_{x}$ is given by $\tau_{n u}$ up to the temperature at which $\tau_{n u}$ intersects Avrami's expression. Obviously as one moves to larger system sizes the intersect moves to lower supercooling (i.e higher temperatures) and in the thermodynamic limit, Avrami's expression is valid for all temperatures. However this is certainly not the case for finite size systems. Notice also that due to the finite size effects small systems gain an extra stability with respect to freezing (i.e more time is needed to freeze the system). One could state that crystallization is controlled by nucleation when
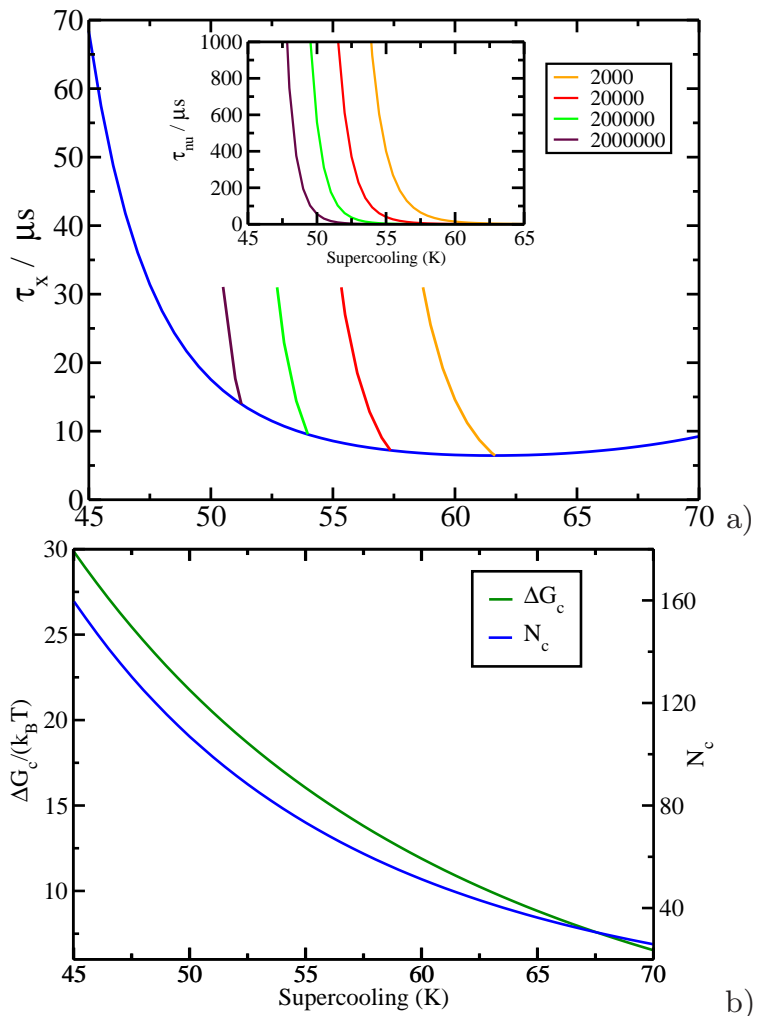

FIG. 9. (a): $\tau_{x}$ for $\phi=0.7$ for the TIP4P $/ 2005$ model as a function of the supercooling. $\tau_{x}$ is the time necessary to crystallize $70 \%$ of the system. Inset: plot of the nucleation time, $\tau_{n u}$, versus the supercooling. (b): Free energy barrier for nucleation and size of the critical cluster for TIP4P/2005 as a function of the supercooling.

$\tau_{n u}$ is much larger than $\tau_{\text {growth }}$, and by ice growth when the opposite is true. This behavior is sensitive to the system size used in the simulations.

After the previous discussion it is clear that the conditions where spontaneous crystallization of TIP4P/2005 water could be most affordable in terms of CPU time would be a system of about 20000 molecules at about $195 \mathrm{~K}$ (i.e $57 \mathrm{~K}$ below the melting point). Under these conditions Avrami's expression is valid and from our estimates it should take about 6 microseconds to freeze the system. That may explain why no ice formation was observed in runs of about one microsecond in previous work 27, 86.

Regarding the possible existence of a liquid-liquid critical point, a key question is to know if the liquid can be equilibrated before it freezes $19,87,90]$. In the case of the TIP4P/2005 model this is equivalent studiying whether 6 microseconds are enough to obtain the properties of metastable water at high supercoolings (i.e in the range of 50 to $65 \mathrm{~K}$ at 1 bar below the melting temperature). Obviously the 6 microseconds refers to the study of this work (i.e at $\mathrm{p}=1 \mathrm{bar}$ ). Further work is needed to analyze how $\tau_{x}$ changes with pressure.

In fig 9 the free energy barrier and size of the critical 
cluster are shown for TIP4P/2005 as a function of the supercooling. Under the conditions where the crystallization time from Avrami's expression is at a minimum we estimate a free energy barrier of $14 k_{B} T$ and a size of the critical cluster of about 60 molecules.

\section{CONCLUSIONS}

In this work we have determined the temperature at which several clusters become critical for both TIP4P and $\mathrm{mW}$ water models. In our previous work 24] we performed similar calculations for TIP4P/2005 and TIP4P/ICE. By assuming that CNT can be used to describe the critical cluster size, the value of the interfacial Ih-water free energy $\gamma$ was obtained. We performed runs of the time evolution of the cluster size with time from its critical value and at the temperature at which it is critical to determine the attachment rate $f^{+}$. Finally the value of the nucleation rate was estimated as a function of the supercooling, by using CNT to estimate the free energy barrier, and the attachment rate to obtain the kinetic prefactor. The main conclusions of our work are:

1. $\gamma$ was found to decrease with temperature with a slope (related to the excess interfacial entropy) of about $-0.25 \mathrm{mN} /(\mathrm{K} . \mathrm{m})$ in reasonable agreement with the previous estimate of Reinhard and Doye for the TIP4P/2005 model (i.e $-0.18 \mathrm{mN} /(\mathrm{K} . \mathrm{m})$ ). For the $\mathrm{mW}$ the temperature dependence was found to be weaker.

2. By extrapolating to the melting temperature an estimate of the interfacial free energy for the planar interface was obtained for several water models. The values of $\gamma$ for the planar interface decrease in the order TIP4P/ICE, mW, TIP4P/2005 and TIP4P. The values obtained of $\gamma$ for the planar interface are in reasonable agreement with the reported experimental values $25-35 \mathrm{mN} / \mathrm{m}$.

3. The attachment rate can be estimated quite well by using the diffusion coefficient, and assuming a typical attachment length of about one molecular diameter (i.e $3.5 \AA$ ). For the mW model, the decrease of $\mathrm{D}$ with $\mathrm{T}$ is weak, certainly accelerating significantly the dynamics at very low temperatures.

4. By fitting the diffusion coefficient to an Arrhenius expression and assuming a linear variation of $\gamma$ with temperature we have estimated $\mathrm{J}$ for a wide range of temperatures. For the $\mathrm{mW}$ the values obtained for $\mathrm{J}$ are in reasonable agreement with previous estimates. The predictions of the TIP4P/2005 for $\mathrm{J}$ are consistent (taking into account the uncertainties) with the experimental values. The model predicts a homogeneous nucleation temperature of about $37 \mathrm{~K}$, in agreement with experiments.

5. At $T_{H}^{\exp }$ the kinetic prefactor to be used in CNT should be of the order of $10^{37}\left(\mathrm{~m}^{-3} \mathrm{~s}^{-1}\right)$ whereas the free energy barrier $\Delta G_{c}$ is of about $53 k_{B} T$. At $T_{H}^{s i m}, \Delta G_{c}$ is of about $14 k_{B} T$.

6. The growth of ice is not arrested at least for temperatures up to $50 \mathrm{~K}$ below the melting point. By using Avrami's equation we estimated that for large systems (i.e large enough to have at least one critical cluster in the simulation box) about 6 microseconds would be required to have a significant fraction of ice for a supercooling of about $60 \mathrm{~K}$. For smaller systems the time would be larger as one needs to wait until a critical cluster is formed. Thus, for small systems, the liquid phase gains kinetic stability so it becomes possible to have the liquid as metastable phase for longer times.

We recognize that the picture provided in this work is far from complete, since we are using a number of approximations in the entire formulation. However it provides an initial framework for forthcoming studies possibly using more sophisticated methods such as umbrella sampling, forward flux sampling or transition path sampling. [91] These calculations will be of much interest, but certainly not cheap from a computational point of view. Although nucleation studies of ice from simulation are still in its infancy we hope our work will encourage further interest in this area, highly relevant for cryopreservation 92], the food industry 93] and climate prediction 94 96.

\section{ACKNOWLEDGEMENTS}

This work was funded by grants FIS2013/43209$\mathrm{P}$ of the MEC, and by the Marie Curie Integration Grants 303941-ANISOKINEQ-FP7-PEOPLE-2011CIG and 322326- COSAAC-FP7-PEOPLE-2012-CIG . C.Valeriani acknowledges financial support from a Juan de La Cierva and E.S. from a Ramon y Cajal Fellowship, respectively. Calculations were carried out thank to the supercomputer facility Tirant from the Spanish Supercomputing Network (RES) (through project QCM-20141-0021). The authors thank Dr. Philip Geiger and Prof. Christoph Dellago for having kindly shared with them configurations of the TIP4P/ICE. We thank Prof. Molinero for providing us the input files to perform runs of the mW model of water using LAMMPS. We thank the two reviewers of this paper for their helpful comments. We thank Dr. Carl McBride for a critical reading of the manuscript. 
[1] K. F. Kelton, Crystal Nucleation in Liquids and Glasses, Vol. 45 (Academic Press, Boston, 1991) p. 75

[2] H. R. Pruppacher, J. Atmosph. Sci. 52, 1924 (1995)

[3] P. Taborek, Phys. Rev. B 32, 5902 (1985)

[4] P. Stockel, I. M. Weldinger, H. Baumgartel, and T. Leisner, J. Phys. Chem. A 109, 2540 (2005)

[5] B. J. Murray, S. L. Broadley, T. W. Wilson, S. J. Bull, R. H. Wills, H. K. Christenson, and E. J. Murray, Phys. Chem. Chem. Phys. 12, 10380 (2010)

[6] L. Granasy, T. Pusztai, and P. F. James, J. Chem. Phys. 117, 6157 (2002)

[7] R. L. Davidchack, R. Handel, J. Anwar, and A. V. Brukhno, J. Chem. Theory Comput. 8, 2383 (2012)

[8] R. Handel, R. L. Davidchack, J. Anwar, and A. Brukhno, Phys. Rev. Lett. 100, 036104 (2008)

[9] C. Vega, E. Sanz, and J. L. F. Abascal, J. Chem. Phys. 122, 114507 (2005)

[10] M. Matsumoto, S. Saito, and I. Ohmine, Nature 416, 409 (2002)

[11] W. L. Jorgensen, J. Chandrasekhar, J. D. Madura, R. W. Impey, and M. L. Klein, J. Chem. Phys. 79, 926 (1983)

[12] R. Radhakrishnan and B. L. Trout, Phys. Rev. Lett. 90, 158301 (2003)

[13] D. Quigley and P. M. Rodger, J. Chem. Phys. 128, 154518 (2008)

[14] A. V. Brukhno, J. Anwar, R. Davidchack, and R. Handel, J. Phys. Condens. Matter 20, 494243 (2008)

[15] V. Molinero and E. B. Moore, The Journal of Physical Chemistry B 113, 4008 (2009)

[16] T. Li, D. Donadio, G. Russo, and G. Galli, Phys. Chem. Chem. Phys. 13, 19807 (2011)

[17] A. Reinhardt and J. P. K. Doye, J. Chem. Phys. 136, 054501 (2012)

[18] L. Filion, M. Hermes, R. Ni, and M. Dijkstra, J. Chem. Phys. 133, 244115 (2010)

[19] E. B. Moore and V. Molinero, Nature 479, 506 (2011)

[20] J. L. F. Abascal and C. Vega, J. Chem. Phys. 123, 234505 (2005)

[21] J. L. F. Abascal, E. Sanz, R. G. Fernandez, and C. Vega, J. Chem. Phys. 122, 234511 (2005)

[22] X.-M. Bai and M. Li, J. Chem. Phys. 122, 224510 (2005)

[23] X.-M. Bai and M. Li, J. Chem. Phys. 124, 124707 (2006)

[24] E. Sanz, C. Vega, J. R. Espinosa, R. Caballero-Bernal, J. L. F. Abascal, and C. Valeriani, J. Am. Chem. Soc. 135, 15008 (2013)

[25] J. L. F. Abascal and C. Vega, J. Chem. Phys. 133, 234502 (2010)

[26] J. L. F. Abascal and C. Vega, J. Chem. Phys. 134, 186101 (2011)

[27] F. Bresme, J. W. Biddle, J. V. Sengers, and M. A. Anisimov, J. Chem. Phys. 140, 161104 (2014)

[28] D. T. Limmer and D. Chandler, J. Chem. Phys. 138, 214504 (2013)

[29] S. Auer and D. Frenkel, J. Chem. Phys. 120, 3015 (2004)

[30] S. Auer and D. Frenkel, Nature 409, 1020 (2001)

[31] C. Valeriani, E. Sanz, and D. Frenkel, J. Chem. Phys. 122, 194501 (2005)

[32] R. G. Pereyra, I. Szleifer, and M. A. Carignano, J. Chem. Phys. 135, 034508 (2011)

[33] B. C. Knott, V. Molinero, M. F. Doherty, and B. Peters, J. Am. Chem. Soc. 134, 19544 (2012)
[34] R. L. Davidchack, J. R. Morris, and B. B. Laird, J. Chem. Phys. 125, 094710 (2006)

[35] R. L. Davidchack, J. Chem. Phys. 133, 234701 (2010)

[36] R. L. Davidchack and B. B. Laird, J. Chem. Phys. 118, 7651 (2003)

[37] W. Lechner and C. Dellago, J. Chem. Phys. 129, 114707 (2008)

[38] M. A. Carignano, J. Phys. Chem. C 111, 501 (2007)

[39] M. Seo, E. Yang, K. Kim, S. Choi, and J. S. Kim, J. Chem. Phys. 137, 154503 (2012)

[40] E. B. Moore and V. Molinero, Phys. Chem. Chem. Phys. 13, 20008 (2011)

[41] W. F. Kuhs, C. Sippel, A. Falenty, and T. C. Hansen, Proceedings of the National Academy of Sciences 109, 21259 (2012)

[42] T. L. Malkin, B. J. Murray, A. V. Brukhno, J. Anwar, and C. G. Salzmann, Proceedings of the National Academy of Sciences 109, 1041 (2012)

[43] J. Benet, L. G. MacDowell, and E. Sanz, Phys. Chem. Chem. Phys. in press, DOI: 10.1039/C4CP03398A (2014)

[44] M. M. Conde and C. Vega, unpublished results $\mathbf{x x}, \mathbf{x x x}$ (2014)

[45] A. Zaragoza, J. R. Espinosa, C. Vega, C. Valeriani, and E. Sanz, unpublished results xx, xxx (2014)

[46] E. Lindahl, B. Hess, and D. van der Spoel, J. Mol. Model. 7, $306(2001)$

[47] G. Bussi, D. Donadio, and M. Parrinello, J. Chem. Phys. 126, 014101 (2007)

[48] M. Parrinello and A. Rahman, J. App. Phys. 52, 7182 (1981)

[49] D. R. Wheeler and J. Newman, Chem. Phys. Lett. 366, 537 (2002)

[50] V. Molinero and E. B. Moore, J. Phys. Chem. B 113, 4008 (2009)

[51] S. Plimpton, J. Comput. Phys. 117, 1 (1995)

[52] C. McBride, C. Vega, E. G. Noya, R. Ramirez, and L. M. Sese, J. Chem. Phys. 131, 024506 (2009)

[53] C. Vega, M. M. Conde, C. McBride, J. L. F. Abascal, E. G. Noya, R. Ramirez, and L. M. Sese, J. Chem. Phys. 132, 046101 (2010)

[54] C. McBride, E. Noya, J.L.Aragones, M. Conde, and C.Vega, Phys. Chem. Chem. Phys. 14, 10140 (2012)

[55] C. McBride, J. Aragones, E.G.Noya, and C. Vega, Phys. Chem. Chem. Phys. 14, 15199 (2012)

[56] C. Vega, J. L. F. Abascal, M. M. Conde, and J. L. Aragones, Faraday Discuss. 141, 251 (2009)

[57] C. Vega and J. L. F. Abascal, Phys. Chem. Chem. Phys 13, 19663 (2011)

[58] R. Feistel and W. Wagner, J. Phys. Chem. Reference Data 35, 1021 (2006)

[59] See EPAPS Document No. E-JCPS for further details about the runs used to determine $N_{c}$ and the attachement rate $f^{+}$.

[60] C. P. Herrero and R. Ramirez, J. Phys. Condens. Matter 26, 233201 (2014)

[61] P. Kumar, S. V. Buldyrev, S. R. Becker, P. H. Poole, F. W. Starr, and H. E. Stanley, Proc. Nat. Acad. Sci. 104, 9575 (2007)

[62] A. Reinhardt and J. P. K. Doye, J. Chem. Phys. 139, 096102 (2013) 
[63] D. Turnbull, Appl. Phys. 21, 1022 (1950)

[64] B. B. Laird, J. Chem. Phys. 115, 2887 (2001)

[65] H. W. Horn, W. C. Swope, J. W. Pitera, J. D. Madura, T. J. Dick, G. L. Hura, and T. Head-Gordon, J. Chem. Phys. 120, 9665 (2004)

[66] S. C. Hardy, Philos. Mag. 35, 471 (1977)

[67] D. Rozmanov and P. G. Kusalik, Phys. Chem. Chem. Phys. 14, 13010 (2012)

[68] D. Rozmanov and P. G. Kusalik, J. Chem. Phys. 136, 044507 (2012)

[69] W. S. Price, H. Ide, and Y. Arata, J. Phys. Chem. 103, 448 (1999)

[70] P. Geiger and C. Dellago, J. Chem. Phys. 139, 164105 (2013)

[71] J. Russo, F. Romano, and H. Tanaka, Nature Materials 13, $733(2014)$

[72] B. J. Murray, S. L. Broadley, T. W. Wilson, S. J. Bull, R. H. Wills, H. K. Christenson, and E. J. Murray, Phys. Chem. Chem. Phys 12, 10380 (2010)

[73] A. Manka, H. Pathak, S. Tanimura, J. Wolk, R. Strey, and B. E. Wyslouzil, Phys. Chem. Chem. Phys 14, 4505 (2012)

[74] C. A. Jeffery and P. H. Austin, J. Geophys. Research 102, 25269 (1997)

[75] J. F. Huang and L. S. Bartell, J. Phys. Chem. 99, 3924 (1995)

[76] L. S. Bartell and J. F. Huang, J. Phys. Chem. 98, 7455 (1994)

[77] V. G. Baidakov, A. O. Tipeev, K. S. Bobrov, and G. V. Ionov, J. Chem. Phys. 132, 234505 (2010)

[78] D. Rozmanov and P. G. Kusalik, Phys. Chem. Chem. Phys 13, 15501 (2011)

[79] H. A. Wilson, Philos. Mag. 50, 238 (1900)

[80] J. Frenkel, Phys. Z. Sowjetunion 1, 498 (1932)

[81] J. Q. Broughton, G. H. Gilmer, and K. A. Jackson, Phys. Rev. Lett. 49, 1496 (1982)

[82] D. Rozmanov and P. G. Kusalik, J. Chem. Phys. 137, 094702 (2012)

[83] M. Avrami, J. Chem. Phys. 7, 1103 (1939)

[84] P. G. Debenedetti, Metastable liquids: Concepts and Principles (Princeton University Press, 1996)

[85] B. A. Berg and S. Dubey, Phys. Rev. Lett. 100, 165702 (2008)

[86] R. Shevchuk and F. Rao, J. Chem. Phys. 137, 036101 (2012)

[87] P. H. Poole, F. Sciortino, U. Essmann, and H. E. Stanley, Nature 360, 324 (1992)

[88] Y. Liu, J. C. Palmer, A. Z. Panagiotopoulos, and P. G. Debenedetti, J. Chem. Phys. 137, 214505 (2012)

[89] D. T. Limmer and D. Chandler, J. Chem. Phys. 135, 134503 (2011)

[90] J. Palmer, F. Martelli, Y. Liu, R. Car, A. Z. Panagiotopoulos, and P. G. Debenedetti, Nature 510, 385 (2014)

[91] P. G. Bolhuis, C. Dellago, P. L. Geissler, and D. Chandler, J. Phys.:Condens. Matter 12, A147 (2000)

[92] G. J. Morris and E. Acton, Cryobiology 66, 85 (2013)

[93] L. R. Maki, E. L. Galyan, C. M.M., and D. R. Caldwell, Appl. Microbiol. 28, 456 (1974)

[94] W. Cantrell and A. Heymsfield, Bull. Amer. Meteor. Soc. 86, 795 (2005)

[95] M. B. Baker, Science 276, 1072 (1997)

[96] P. J. DeMott, A. J. Prenni, X. Liu, S. M. Kreidenweis, M. D. Petters, C. H. Twohy, M. S. Richardson, T. Ei- dhammer, and D. C. Rogers, Proc. Nat. Acad. Sci. USA 107, 11217 (2010) 


\section{Appendix A: Critical cluster}

Here, we show the performed trajectories done for the TIP4P and $\mathrm{mW}$ water models to establish in what degree of supercooling the cluster inserted becomes critical. The performed trajectories for the TIP4P/2005 and TIP4P/ICE water models for the same purpose are shown in the Supplementary material of Ref [E. Sanz, C. Vega, J. R. Espinosa, R. Caballero-Bernal, J. L. F. Abascal and C. Valeriani, JACS, 135, 15008, (2013)].

TABLE V. $N_{t}$ is the total number of molecules in the system (ice cluster + surrounding liquid water molecules) and $N_{c}$ is the number of molecules of the inserted spherical ice cluster after equilibration of the interface.

\begin{tabular}{ccccc}
\hline$N_{t}$ & $N_{c}^{\text {TIP4P/2005 }}$ & $N_{c}^{\text {TIP4P/Ice }}$ & $N_{c}^{\text {TIP4P }}$ & $N_{c}^{m W}$ \\
\hline 22712 & 600 & 600 & 600 & 600 \\
76781 & 3170 & 3167 & 3170 & 3167 \\
182585 & 7931 & 7926 & 7931 & 7926 \\
\hline
\end{tabular}

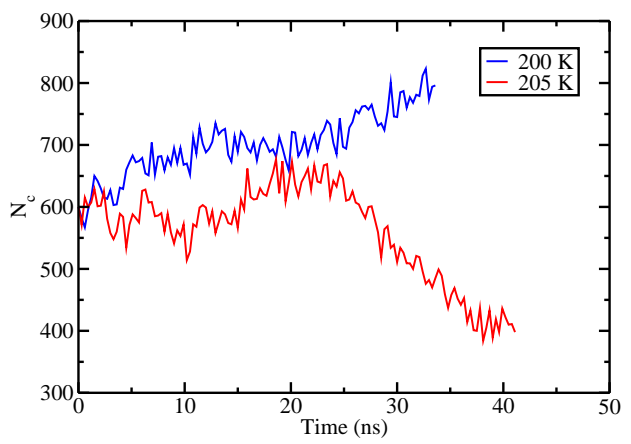

FIG. 10. Performed trajectories for the cluster of 600 ice molecules for the TIP4P model. The temperature for which this cluster is critical is $202.5 \mathrm{~K}$.

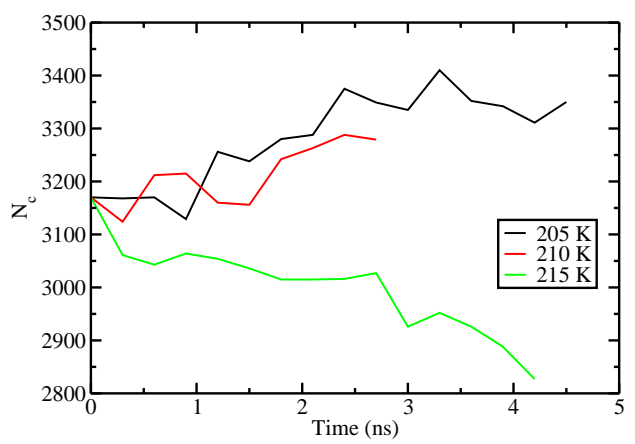

FIG. 11. Performed trajectories for the cluster of 3170 ice molecules for the TIP4P model. For this cluster size, the critical temperature is $212.5 \mathrm{~K}$

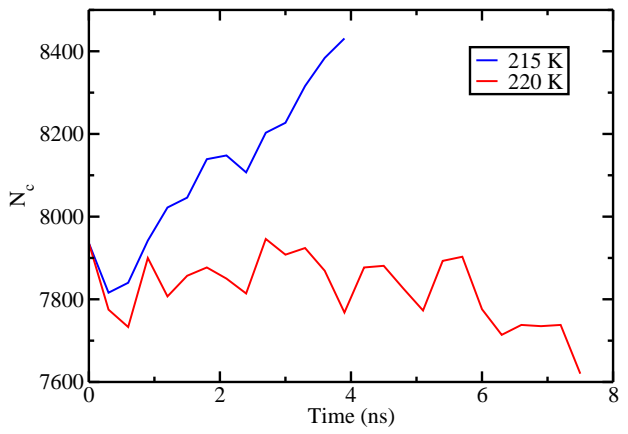

FIG. 12. Performed trajectories for the cluster of 7931 ice molecules for the TIP4P model. In this case the critical temperature is $217.5 \mathrm{~K}$.

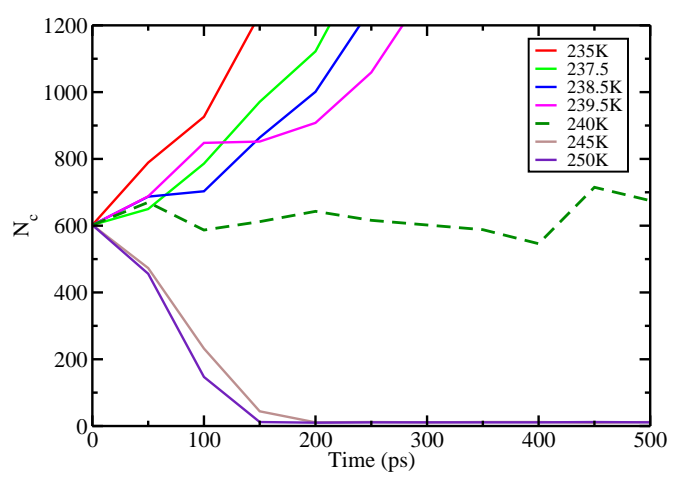

FIG. 13. Performed trajectories for the cluster of 600 ice molecules for the $\mathrm{mW}$ model. The temperature for which this cluster is critical is $240 \mathrm{~K}$.

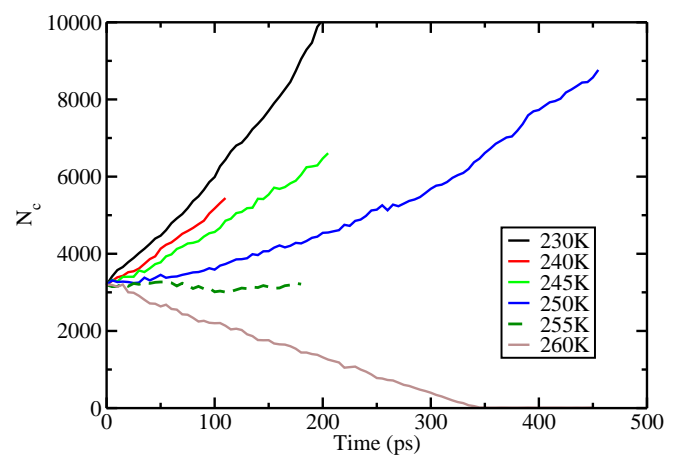

FIG. 14. Performed trajectories for the cluster of 3167 ice molecules for the $\mathrm{mW}$ model whose critical temperature is $255 \mathrm{~K}$. 


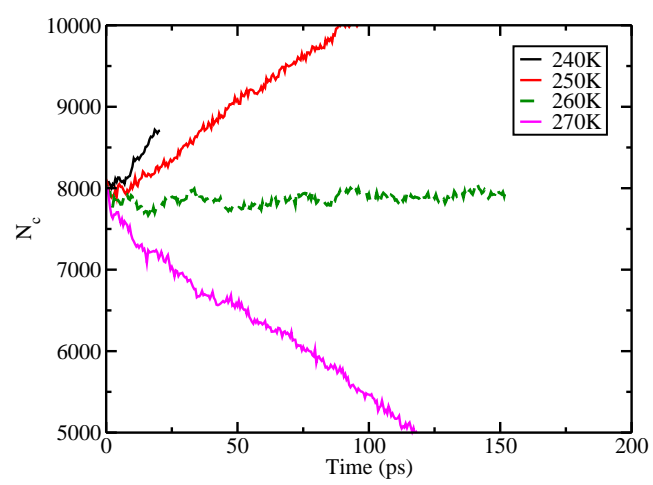

FIG. 15. Performed trajectories for the cluster of 7926 ice molecules for the $\mathrm{mW}$ model. The temperature for which this cluster is critical is $260 \mathrm{~K}$. 
Appendix B: Attachment rates

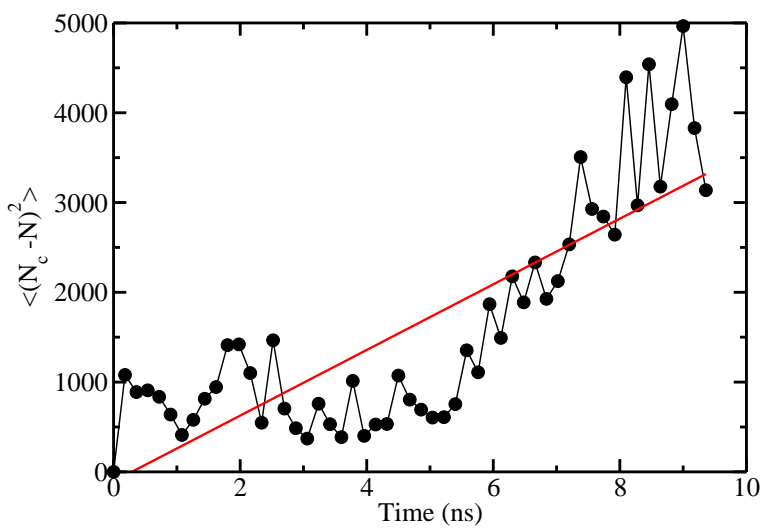

FIG. 16. Attachment rate calculated for the cluster of 600 ice molecules for the TIP4P model. 10 trajectories were performed under conditions of $\mathrm{T}=202.5 \mathrm{~K}$ and $\mathrm{P}=1 \mathrm{bar}$.

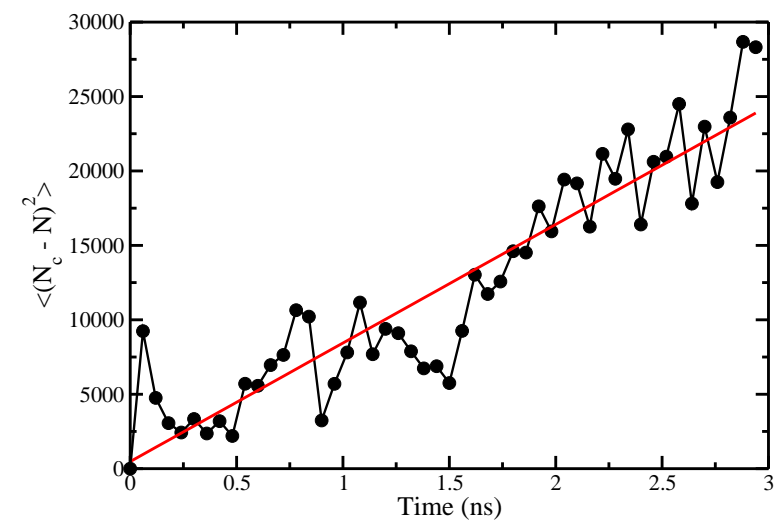

FIG. 17. Attachment rate calculated for the cluster of 3170 ice molecules for the TIP4P model. 10 trajectories were performed under conditions of $\mathrm{T}=212.5 \mathrm{~K}$ and $\mathrm{P}=1 \mathrm{bar}$.

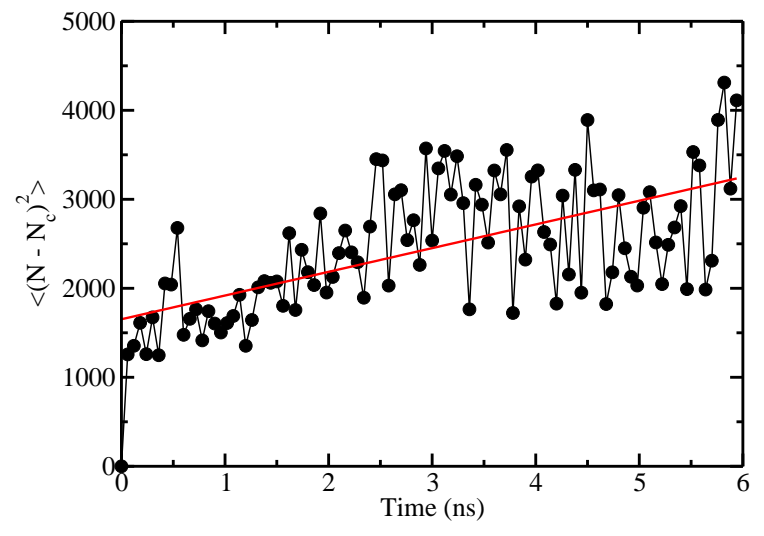

FIG. 18. Attachment rate calculated for the cluster of 600 ice molecules for the TIP4P/2005 model. 10 trajectories were performed under conditions of $\mathrm{T}=222.5 \mathrm{~K}$ and $\mathrm{P}=1 \mathrm{bar}$. 


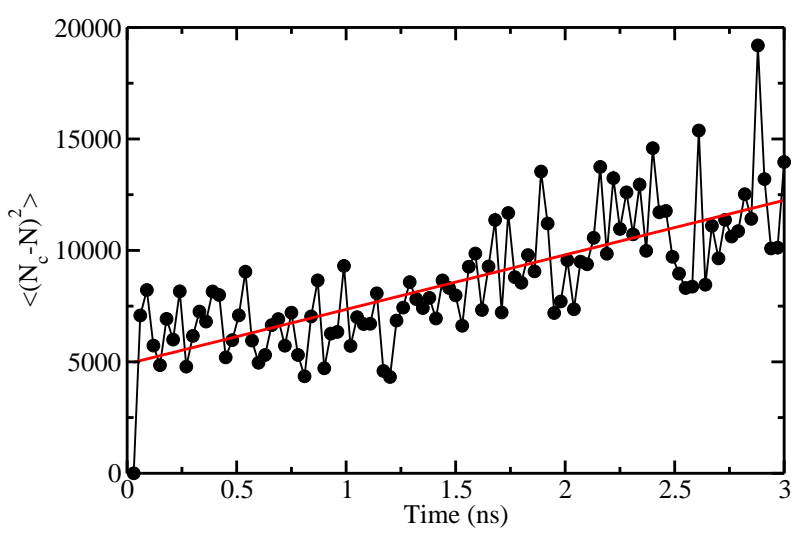

FIG. 19. Attachment rate calculated for the cluster of 3170 ice molecules for the TIP4P/2005 model. 10 trajectories were performed under conditions of $\mathrm{T}=232.5 \mathrm{~K}$ and $\mathrm{P}=1 \mathrm{bar}$.

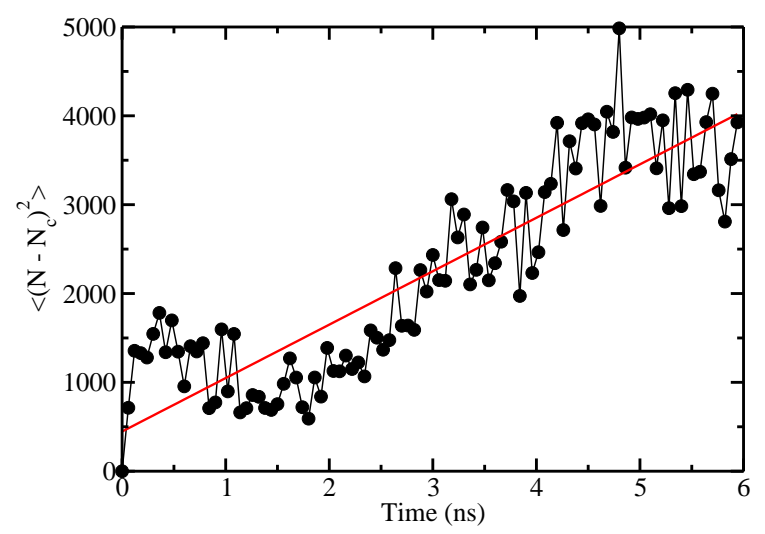

FIG. 20. Attachment rate calculated for the cluster of 600 ice molecules for the TIP4P/ICE model. 10 trajectories were performed under conditions of $\mathrm{T}=237.5 \mathrm{~K}$ and $\mathrm{P}=1 \mathrm{bar}$.

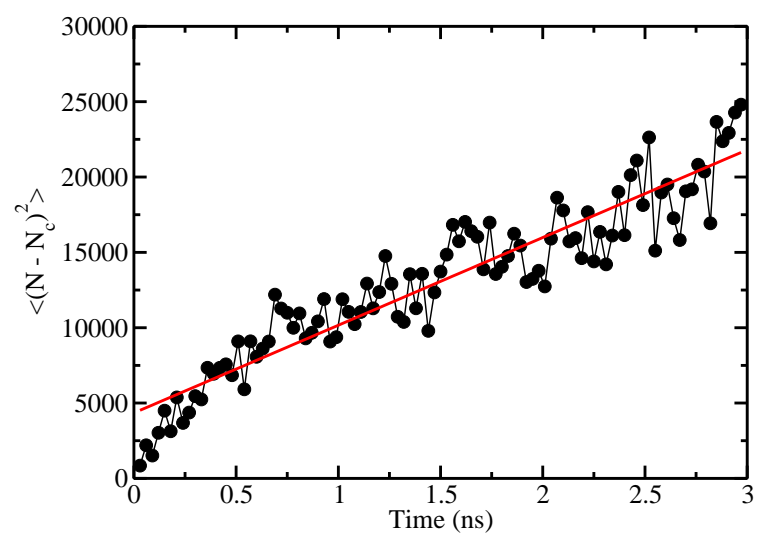

FIG. 21. Attachment rate calculated for the cluster of 3167 ice molecules for the TIP4P/ICE model. 10 trajectories were performed under conditions of $\mathrm{T}=252.5 \mathrm{~K}$ and $\mathrm{P}=1 \mathrm{bar}$. 


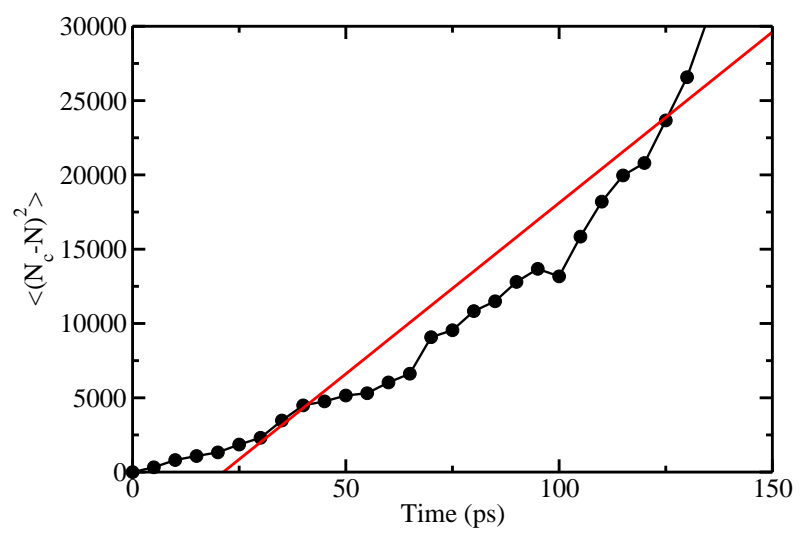

FIG. 22. Attachment rate calculated for the cluster of 600 ice molecules for the $\mathrm{mW}$ model. 30 trajectories were performed under conditions of $\mathrm{T}=240 \mathrm{~K}$ and $\mathrm{P}=1 \mathrm{bar}$.

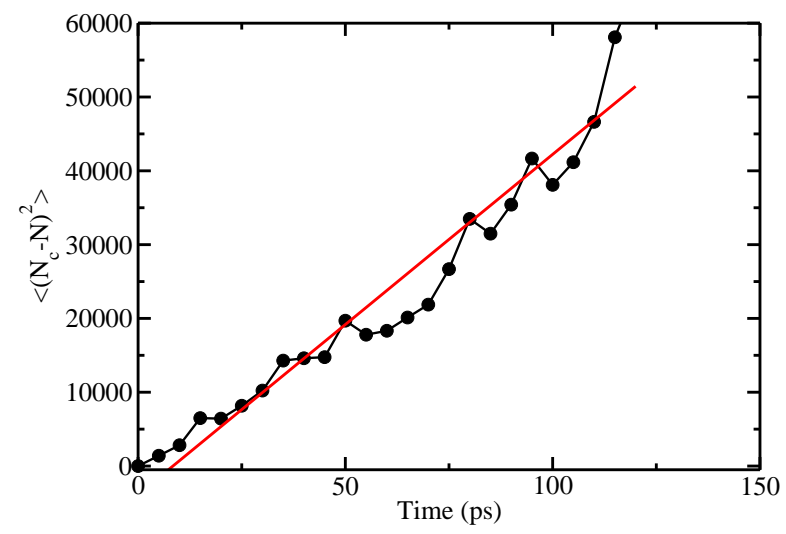

FIG. 23. Attachment rate calculated for the cluster of 3167 ice molecules for the $\mathrm{mW}$ model. 30 trajectories were performed under conditions of $\mathrm{T}=255 \mathrm{~K}$ and $\mathrm{P}=1$ bar.

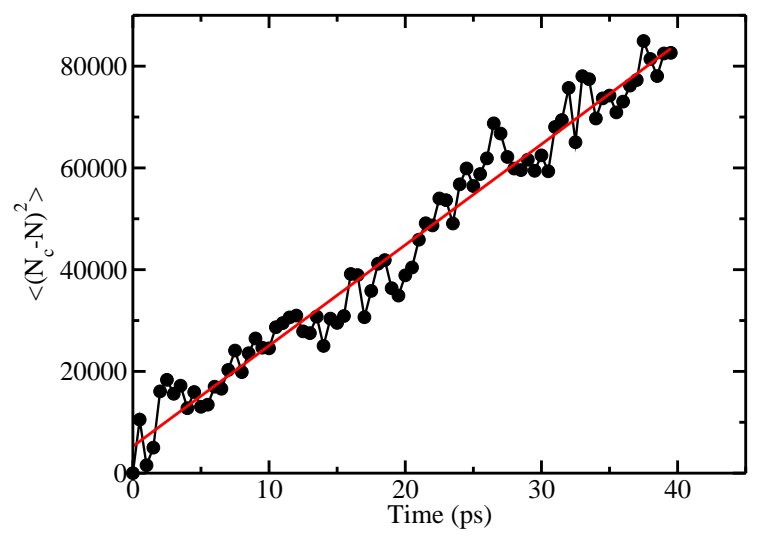

FIG. 24. Attachment rate calculated for the cluster of 7926 ice molecules for the $\mathrm{mW}$ model. 30 trajectories were performed under conditions of $\mathrm{T}=260 \mathrm{~K}$ and $\mathrm{P}=1 \mathrm{bar}$. 